\title{
Regime-Switching Jump Diffusions with Non-Lipschitz Coefficients and Countably Many Switching States: Existence and Uniqueness, Feller, and Strong Feller Properties ${ }^{\star}$
}

Fubao Xi, George Yin, and Chao Zhu

\begin{abstract}
This work focuses on a class of regime-switching jump diffusion processes, which is a two component Markov processes $(X(t), \Lambda(t))$, where $\Lambda(t)$ is a component representing discrete events taking values in a countably infinite set. Considering the corresponding stochastic differential equations, our main focus is on treating those with non-Lipschitz coefficients. We first show that there exists a unique strong solution to the corresponding stochastic differential equation. Then Feller and strong Feller properties are investigated.
\end{abstract}

Keywords. Regime-switching jump diffusion, non-Lipschitz condition, Feller property, strong Feller property.

Mathematics Subject Classification. 60J27, 60J60, 60J75, 60G51.

F. Xi

School of Mathematics and Statistics, Beijing Institute of Technology, Beijing 100081, China, e-mail: xifb@bit.edu.cn

G. Yin

Department of Mathematics, Wayne State University, Detroit, MI 48202, e-mail: gyin@math.wayne.edu

C. Zhu

Department of Mathematical Sciences, University of Wisconsin-Milwaukee, Milwaukee, WI 53201, e-mail: zhu@uwm.edu

* The study was initiated during a workshop held at IMA, University of Minnesota. The support of IMA with funding provided by the National Science Foundation is acknowledged. The research was also supported in part by the National Natural Science Foundation of China under Grant No. 11671034, the US Army Research Office, and the Simons Foundation Collaboration Grant (No. 523736) 


\section{Introduction}

In the past decade, much attention has been devoted to a class of hybrid systems, namely, regime-switching diffusions. Roughly, such processes can be considered as a two component process $(X(t), \Lambda(t))$, an analog (or continuous state) component $X(t)$ and a switching (or discrete event) process $\Lambda(t)$. Some of the representative works can be found in Mao and Yuan (2006) and Yin and Zhu (2010). The former dealt with regime-switching diffusions in which the switching process is a continuous-time Markov chain independent of the Brownian motion, whereas the latter treated processes in which the switching component depends on the continuous-state component. It has been found that the discrete event process, taking values in a finite or countable set, can be used to delineate, for example, random environment or other random factors that are not represented in the usual diffusion formulation. Seemingly similar to the diffusion processes, in fact, regime-switching diffusions have very different behavior compared to the usual diffusion processes. For example, it has been demonstrated in Lawley et al. (2014), Yin et al. (2012) that two stable (resp., unstable) ordinary differential equations can be coupled to produce an unstable (resp., stable) regime-switching system. The consideration of regime-switching diffusions has substantially enlarged the applicability of stochastic processes for a wide variety of problems ranging from network systems, multiagent systems, ecological and biological applications, financial engineering, risk management, etc.

Continuing on the effort of studying regime-switching diffusions, Chen et al. (2018a) obtained maximum principle and Harnack inequalities for switching jump diffusions using mainly probabilistic arguments, and Chen el al. (2018b) proceeded further to obtain recurrence and ergodicity of switching jump diffusions. In another direction, $\mathrm{Xi}$ and Zhu (2017) dealt with regime-switching jump diffusions with countable number of switching values. Nguyen and Yin (2016) considered switching diffusions in which the switching process depends on the past information of the continuous state and takes values in a countable state space; the corresponding recurrence and ergodicity was considered in Nguyen and Yin (2018).

A standing assumption in the aforementioned references is that the coefficients of the associated stochastic differential equations are (locally) Lipschitz. While it is a convenient assumption, it is rather restrictive in many applications. For example, the diffusion coefficients in the Feller branching diffusion and the Cox-IngersollRoss model are only Hölder continuous. We refer to Chapters 12 and 13 of Klebaner (2005) for an introduction to these models. Motivated by these considerations, there has been much efforts devoted to the study of stochastic differential equations with non-Lipschitz coefficients. An incomplete list includes Bass (2003), Fang and Zhang (2005), Li and Mytnik (2011), Li and Pu (2012), Yamada and Watanabe (1971), among many others.

While there are many works on diffusions and jump diffusions with non-Lipschitz coefficients, the related research on regime-switching jump diffusions is relatively scarce. This work aims to investigate regime-switching jump diffusion processes with non-Lipschitz coefficients. More precisely, the purpose of this paper is two- 
fold: (i) to establish the strong existence and uniqueness result for stochastic differential equations associated with regime-switching jump diffusions, in which the coefficients are non-Lipschitz and the switching component has countably many states; and (ii) to derive sufficient conditions for Feller and strong Feller properties. Our focus is devoted to establishing non-Lipschitz sufficient conditions for the aforementioned properties.

The rest of the paper is arranged as follows. Examining the associated stochastic differential equations, we begin to obtain the existence and uniqueness of the solution of the stochastic differential equations in Section 2. Then Section 3 proceeds with the study of Feller properties. Section 4 further extends the study to treat strong Feller properties.

\section{Strong Solution: Existence and Uniqueness}

We work with $(U, \mathfrak{U})$ a measurable space, $v$ a $\sigma$-finite measure on $U$, and $\mathbb{S}:=$ $\{1,2, \ldots\}$. Assume that $d \geq 1$ is a positive integer, $b: \mathbb{R}^{d} \times \mathbb{S} \mapsto \mathbb{R}^{d}, \sigma: \mathbb{R}^{d} \times \mathbb{S} \mapsto$ $\mathbb{R}^{d \times d}$, and $c: \mathbb{R}^{d} \times \mathbb{S} \times U \mapsto \mathbb{R}^{d}$ be Borel measurable functions. Let $(X, \Lambda)$ be a right continuous, strong Markov process with left-hand limits on $\mathbb{R}^{d} \times \mathbb{S}$. The first component $X$ satisfies the following stochastic differential-integral equation

$$
\mathrm{d} X(t)=b(X(t), \Lambda(t)) \mathrm{d} t+\sigma(X(t), \Lambda(t)) \mathrm{d} W(t)+\int_{U} c(X(t-), \Lambda(t-), u) \widetilde{N}(\mathrm{~d} t, \mathrm{~d} u),
$$

where $W$ is a standard $d$-dimensional Brownian motion, $N$ is a Poisson random measure on $[0, \infty) \times U$ with intensity $\mathrm{d} t v(\mathrm{~d} u)$, and $\widetilde{N}$ is the associated compensated Poisson random measure. The second component $\Lambda$ is a continuous-time random process taking values in the countably infinite set $\mathbb{S}$ such that

$$
\mathbb{P}\{\Lambda(t+\Delta)=l \mid \Lambda(t)=k, X(t)=x\}= \begin{cases}q_{k l}(x) \Delta+o(\Delta), & \text { if } k \neq l, \\ 1+q_{k k}(x) \Delta+o(\Delta), & \text { if } k=l,\end{cases}
$$

uniformly in $\mathbb{R}^{d}$, provided $\Delta \downarrow 0$.

To proceed, we construct a family of disjoint intervals $\left\{\Delta_{i j}(x): i, j \in \mathbb{S}\right\}$ on the positive half real line as follows

$$
\begin{aligned}
\Delta_{12}(x) & =\left[0, q_{12}(x)\right), \\
\Delta_{13}(x) & \left.=\left[q_{12}(x)\right), q_{12}(x)+q_{13}(x)\right), \\
& \vdots \\
\Delta_{21}(x) & =\left[q_{1}(x), q_{1}(x)+q_{21}(x)\right), \\
\Delta_{23}(x) & \left.=\left[q_{1}(x)+q_{21}(x)\right), q_{1}(x)+q_{21}(x)+q_{23}(x)\right),
\end{aligned}
$$




$$
\Delta_{31}(x)=\left[q_{1}(x)+q_{2}(x), q_{1}(x)+q_{2}(x)+q_{31}(x)\right),
$$

where for convenience, we set $\Delta_{i j}(x)=\emptyset$ if $q_{i j}(x)=0, i \neq j$. Note that for each $x \in \mathbb{R}^{d},\left\{\Delta_{i j}(x): i, j \in \mathbb{S}\right\}$ are disjoint intervals, and the length of the interval $\Delta_{i j}(x)$ is equal to $q_{i j}(x)$. We then define a function $h: \mathbb{R}^{d} \times \mathbb{S} \times \mathbb{R}_{+} \rightarrow \mathbb{R}$ by

$$
h(x, k, r)=\sum_{l \in \mathbb{S}}(l-k) \mathbf{1}_{\Delta_{k l}(x)}(r) .
$$

That is, for each $x \in \mathbb{R}^{d}$ and $k \in \mathbb{S}$, we set $h(x, k, r)=l-k$ if $r \in \Delta_{k l}(x)$ for some $l \neq k$; otherwise $h(x, k, r)=0$. Consequently, we can describe the evolution of $\Lambda$ using the following stochastic differential equation

$$
\Lambda(t)=\Lambda(0)+\int_{0}^{t} \int_{\mathbb{R}_{+}} h(X(s-), \Lambda(s-), r) N_{1}(\mathrm{~d} s, \mathrm{~d} r),
$$

where $N_{1}$ is a Poisson random measure on $[0, \infty) \times[0, \infty)$ with characteristic measure $\mathfrak{m}(\mathrm{d} z)$, the Lebesgue measure.

For convenience in the subsequent discussion, let us give the infinitesimal generator $\mathscr{A}$ of the regime-switching jump diffusion $(X, \Lambda)$

$$
\mathscr{A} f(x, k):=\mathscr{L}_{k} f(x, k)+Q(x) f(x, k),
$$

with $a(x, k):=\sigma \sigma^{T}(x, k)$ and

$$
\begin{aligned}
\mathscr{L}_{k} f(x, k):= & \frac{1}{2} \operatorname{tr}\left(a(x, k) \nabla^{2} f(x, k)\right)+\langle b(x, k), \nabla f(x, k)\rangle \\
& +\int_{U}(f(x+c(x, k, u), k)-f(x, k)-\langle\nabla f(x, k), c(x, k, u)\rangle) v(\mathrm{~d} u), \\
Q(x) f(x, k):= & \sum_{j \in \mathbb{S}} q_{k j}(x)[f(x, j)-f(x, k)] \\
= & \int_{[0, \infty)}[f(x, k+h(x, k, z))-f(x, k)] \mathfrak{m}(\mathrm{d} z) .
\end{aligned}
$$

Define a metric $\lambda(\cdot, \cdot)$ on $\mathbb{R}^{d} \times \mathbb{S}$ as $\lambda((x, m),(y, n))=|x-y|+d(m, n)$, where $d(m, n)=\mathbf{1}_{\{m \neq n\}}$ is the discrete metric on $\mathbb{S}$. Let $\mathfrak{B}\left(\mathbb{R}^{d} \times \mathbb{S}\right)$ be the Borel $\sigma$-algebra on $\mathbb{R}^{d} \times \mathbb{S}$. Then $\left(\mathbb{R}^{d} \times \mathbb{S}, \lambda(\cdot, \cdot), \mathfrak{B}\left(\mathbb{R}^{d} \times \mathbb{S}\right)\right)$ is a locally compact and separable metric space. For the existence and uniqueness of the strong Markov process $(X, \Lambda)$ satisfying system (1) and (4), we make the following assumptions.

Assumption 2.1 There exists a nondecreasing function $\zeta:[0, \infty) \mapsto[1, \infty)$ that is continuously differentiable and that satisfies

$$
\int_{0}^{\infty} \frac{\mathrm{d} r}{r \zeta(r)+1}=\infty
$$


such that for all $x \in \mathbb{R}^{d}$ and $k \in \mathbb{S}$,

$$
\begin{aligned}
& 2\langle x, b(x, k)\rangle+|\sigma(x, k)|^{2}+\int_{U}|c(x, k, u)|^{2} v(\mathrm{~d} u) \leq H\left[|x|^{2} \zeta\left(|x|^{2}\right)+1\right], \\
& q_{k}(x):=-q_{k k}(x)=\sum_{l \in \mathbb{S} \backslash\{k\}} q_{k l}(x) \leq H k, \\
& \sum_{l \in \mathbb{S} \backslash\{k\}}(f(l)-f(k)) q_{k l}(x) \leq H(1+\Phi(x)+f(k)),
\end{aligned}
$$

where $H$ is a positive constant,

$$
\Phi(x):=\exp \left\{\int_{0}^{|x|^{2}} \frac{\mathrm{d} r}{r \zeta(r)+1}\right\}, \quad x \in \mathbb{R}^{d},
$$

and the function $f: \mathbb{S} \mapsto \mathbb{R}_{+}$is nondecreasing satisfying $f(m) \rightarrow \infty$ as $m \rightarrow \infty$. In addition, assume there exists some $\delta \in(0,1]$ such that

$$
\sum_{l \in \mathbb{S} \backslash\{k\}}\left|q_{k l}(x)-q_{k l}(y)\right| \leq H|x-y|^{\delta}
$$

for all $k \in \mathbb{S}$ and $x, y \in \mathbb{R}^{d}$.

Assumption 2.2 Assume the following conditions hold.

- If $d=1$, then there exist a positive number $\delta_{0}$ and a nondecreasing and concave function $\rho:[0, \infty) \mapsto[0, \infty)$ satisfying

$$
\int_{0^{+}} \frac{\mathrm{d} r}{\rho(r)}=\infty
$$

such that for all $k \in \mathbb{S}, R>0$, and $x, z \in \mathbb{R}$ with $|x| \vee|z| \leq R$ and $|x-z| \leq \delta_{0}$,

$$
\begin{aligned}
& \operatorname{sgn}(x-z)(b(x, k)-b(z, k)) \leq \kappa_{R} \rho(|x-z|), \\
& |\sigma(x, k)-\sigma(z, k)|^{2}+\int_{U}|c(x, k, u)-c(z, k, u)|^{2} v(\mathrm{~d} u) \leq \kappa_{R}|x-z|,
\end{aligned}
$$

where $\kappa_{R}$ is a positive constant and $\operatorname{sgn}(a)=1$ if $a>0$ and -1 if $a \leq 0$. In addition, for each $k \in \mathbb{S}$, the function $c$ satisfies that

$$
\text { the function } x \mapsto x+c(x, k, u) \text { is nondecreasing for all } u \in U \text {; }
$$

or, there exists some $\beta>0$ such that

$$
|x-z+\theta(c(x, k, u)-c(z, k, u))| \geq \beta|x-z|, \quad \forall(x, z, u, \theta) \in \mathbb{R} \times \mathbb{R} \times U \times[0,1] .
$$

- If $d \geq 2$, then there exist a positive number $\delta_{0}$, and a nondecreasing and concave function $\rho:[0, \infty) \mapsto[0, \infty)$ satisfying 


$$
0<\rho(r) \leq(1+r)^{2} \rho(r /(1+r)) \text { for all } r>0, \text { and } \int_{0^{+}} \frac{\mathrm{d} r}{\rho(r)}=\infty
$$

such that for all $k \in \mathbb{S}, R>0$, and $x, z \in \mathbb{R}^{d}$ with $|x| \vee|z| \leq R$ and $|x-z| \leq \delta_{0}$,

$$
\begin{aligned}
& 2\langle x-z, b(x, k)-b(z, k)\rangle+|\sigma(x, k)-\sigma(z, k)|^{2} \\
& \quad+\int_{U}|c(x, k, u)-c(z, k, u)|^{2} v(\mathrm{~d} u) \leq \kappa_{R} \rho\left(|x-z|^{2}\right),
\end{aligned}
$$

where $\kappa_{R}$ is a positive constant.

Remark 2.3 We make some comments concerning Assumptions 2.1 and 2.2. Examples of functions satisfying (8) include $\zeta(r)=1, \zeta(r)=\log r$, and $\zeta(r)=$ $\log r \log (\log r)$ for $r$ large. When $\zeta(r)=1$, (9) reduces to the usual linear growth condition. With other choices of $\zeta$, (8) allows super-linear condition for the coefficients of (1) with respect to the variable $x$ for each $k \in \mathbb{S}$. This is motivated by applications such as Lotka-Volterra models, in which the coefficients have superlinear growth conditions. Conditions (10) and (11) are imposed so that the $\Lambda$ component will not explode in finite time with probability 1 ; see the proof of Theorem 2.5 for details.

Examples of functions satisfying (14) or (19) include $\rho(r)=r$ and concave and increasing functions such as $\rho(r)=r \log (1 / r), \rho(r)=r \log (\log (1 / r))$, and $\rho(r)=r \log (1 / r) \log (\log (1 / r))$ for $r \in(0, \delta)$ with $\delta>0$ small enough. When $\rho(r)=r$, Assumption 2.2 is just the usual local Lipschitz condition. With other choices of continuity modularity, Assumption 2.2 allows the drift, diffusion, and jump coefficients of (1) to be non-Lipschitz with respect to the variable $x$. This, in turn, presents more opportunities for building realistic and flexible mathematical models for a wide range of applications. Indeed, non-Lipschitz coefficients are present in areas such as branching diffusion in biology, the Cox-Ingersoll-Ross model in math finance, etc.

It is also worth pointing out that (15), (16), and (20) of Assumption 2.2 only require the modulus of continuity to hold in a small neighborhood of the diagonal line $x=z$ in $\mathbb{R}^{d} \otimes \mathbb{R}^{d}$ with $|x| \vee|z| \leq R$ for each $R>0$. This is in contrast to those in $\mathrm{Li}$ and $\mathrm{Pu}$ (2012) and adds some subtlety in the proof of pathwise uniqueness for (21).

When $d=1$, Assumption 2.2 allows the diffusion coefficient $\sigma(\cdot, k)$ to be locally Hölder continuous with exponent $\alpha \in\left[\frac{1}{2}, 1\right]$. This is the celebrated result in Yamada and Watanabe (1971). Such a result was extended to stochastic differential equations with jumps; see, for example, Fu and Li (2010), Li and Mytnik (2011) and Li and $\mathrm{Pu}$ (2012), among others. In particular, Li and $\mathrm{Pu}$ (2012) shows that if (17) holds, the function $x \mapsto \int_{U} c(x, k, u) v(\mathrm{~d} u)$ can be locally Hölder continuous with exponent $\alpha \in\left[\frac{1}{2}, 1\right]$ as well. The continuity assumption (15) on the drift coefficient $b(\cdot, k)$ is slightly more general than that in $\mathrm{Li}$ and $\mathrm{Pu}$ (2012). In particular, (15) will be satisfied as long as $b(\cdot, k)$ is decreasing.

Lemma 2.4 Suppose Assumption 2.2 and (9) hold. Then for each $k \in \mathbb{S}$, the stochastic differential equation 


$$
X(t)=x+\int_{0}^{t} b(X(s), k) \mathrm{d} s+\int_{0}^{t} \sigma(X(s), k) \mathrm{d} W(s)+\int_{0}^{t} \int_{U} c(X(s-), k, u) \widetilde{N}(\mathrm{~d} s, \mathrm{~d} u)
$$

has a unique non-explosive strong solution.

Proof. Condition (9) guarantees that the solution to (21) will not explode in finite time with probability 1; see, for example, Theorem 2.1 in Xi and Zhu (2018a). When $d \geq 2$, the existence and uniqueness of a strong solution to (21) under Assumption 2.2 follows from Theorem 2.8 of $\mathrm{Xi}$ and $\mathrm{Zhu}$ (2018a).

When $d=1$, we follow the arguments in the proof of Theorem 3.2 of $\mathrm{Li}$ and $\mathrm{Pu}$ (2012) to show that pathwise uniqueness holds for (21). First, let $\left\{a_{n}\right\}$ be a strictly decreasing sequence of real numbers satisfying $a_{0}=1, \lim _{n \rightarrow \infty} a_{n}=0$, and $\int_{a_{n}}^{a_{n-1}} \frac{\mathrm{d} r}{r}=n$ for each $n \geq 1$. For each $n \geq 1$, let $\rho_{n}$ be a nonnegative continuous function with support on $\left(a_{n}, a_{n-1}\right)$ so that

$$
\int_{a_{n}}^{a_{n-1}} \rho_{n}(r) \mathrm{d} r=1 \text { and } \rho_{n}(r) \leq 2(k r)^{-1} \text { for all } r>0 .
$$

For $x \in \mathbb{R}$, define

$$
\psi_{n}(x)=\int_{0}^{|x|} \int_{0}^{y} \rho_{n}(z) \mathrm{d} z \mathrm{~d} y .
$$

We can immediately verify that $\psi_{n}$ is even and twice continuously differentiable, with

$$
\psi_{n}^{\prime}(r)=\operatorname{sgn}(r) \int_{0}^{|r|} \rho_{n}(z) \mathrm{d} z=\operatorname{sgn}(r)\left|\psi_{n}^{\prime}(r)\right|
$$

and

$$
\left|\psi_{n}^{\prime}(r)\right| \leq 1, \quad 0 \leq|r| \psi_{n}^{\prime \prime}(r)=|r| \rho_{n}(|r|) \leq \frac{2}{n}, \quad \text { and } \quad \lim _{n \rightarrow \infty} \psi_{n}(r)=|r|
$$

for $r \in \mathbb{R}$. Furthermore, for each $r>0$, the sequence $\left\{\psi_{n}(r)\right\}_{n \geq 1}$ is nondecreasing. Note also that for each $n \in \mathbb{N}, \psi_{n}, \psi_{n}^{\prime}$, and $\psi_{n}^{\prime \prime}$ all vanish on the interval $\left(-a_{n}, a_{n}\right)$. Moreover the classical arguments reveal that

$$
\begin{aligned}
& \frac{1}{2} \psi_{n}^{\prime \prime}(x-z)|\sigma(x, k)-\sigma(z, k)|^{2}+\int_{U}\left[\psi_{n}(x-z+c(x, k, u)-c(z, k, u))\right. \\
& \left.-\psi_{n}(x-z)-\psi_{n}^{\prime}(x-z)(c(x, k, u)-c(z, k, u))\right] v(\mathrm{~d} u) \\
& \leq \frac{1}{2} \cdot \frac{2}{n} \kappa_{R}+\frac{\kappa_{R}}{n}\left(\frac{1}{\beta} \vee 2\right) \leq K \frac{\kappa_{R}}{n}
\end{aligned}
$$

for all $x, z$ with $|x| \vee|z| \leq R$ and $0<|x-z| \leq \delta_{0}$, where $K$ is a positive constant independent of $R$ and $n$. On the other hand, for any $x, z \in \mathbb{R}$ with $|x| \vee|z| \leq R$ and $|x-z| \leq \delta_{0}$, it follows from (15) and (23) that

$$
\begin{aligned}
\psi_{n}^{\prime}(x-z)(b(x, k)-b(z, k)) & =\operatorname{sgn}(x-z)\left|\psi_{n}^{\prime}(x-z)\right|(b(x, k)-b(z, k)) \\
& \leq \kappa_{R} \rho(|x-z|)
\end{aligned}
$$


Let $\widetilde{X}$ and $X$ be two solutions to (21). Denote $\Delta_{t}:=\widetilde{X}(t)-X(t)$ for $t \geq 0$. Assume $\left|\Delta_{0}\right|=|\widetilde{x}-x|<\delta_{0}$ and define

$$
S_{\delta_{0}}:=\inf \left\{t \geq 0:\left|\Delta_{t}\right| \geq \delta_{0}\right\}=\inf \left\{t \geq 0:|\widetilde{X}(t)-X(t)| \geq \delta_{0}\right\} .
$$

For $R>0$, let $\tau_{R}:=\inf \{t \geq 0:|\widetilde{X}(t)| \vee|X(t)|>R\}$. Then $\tau_{R} \rightarrow \infty$ a.s. as $R \rightarrow \infty$. Moreover, by Itô's formula, we have

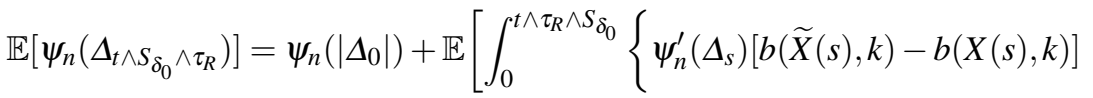

$$
\begin{aligned}
& +\frac{1}{2} \psi_{n}^{\prime \prime}\left(\Delta_{s}\right)[\sigma(\widetilde{X}(s), k)-\sigma(X(s), k)]^{2} \\
& +\int_{U}\left[\psi_{n}\left(\Delta_{s}+c(\widetilde{X}(s), k, u)-c(\widetilde{X}(s), k, u)\right)-\psi_{n}\left(\Delta_{s}\right)\right. \\
& \left.\left.\left.\left.-\psi_{n}^{\prime}\left(\Delta_{s}\right)(c(\widetilde{X}(s), k, u)-c(\widetilde{X}(s), k, u))\right)\right] v(\mathrm{~d} u)\right\} \mathrm{d} s\right] .
\end{aligned}
$$

Furthermore, using (25) and (26), we obtain

$$
\begin{aligned}
\mathbb{E}\left[\psi _ { n } \left(\Delta_{\left.\left.t \wedge S_{\delta_{0}} \wedge \tau_{R}\right)\right]}\right.\right. & \leq \psi_{n}\left(\left|\Delta_{0}\right|\right)+\mathbb{E}\left[\int_{0}^{t \wedge \tau_{R} \wedge S_{\delta_{0}}}\left(\kappa_{R} \rho\left(\left|\Delta_{s}\right|\right)+K \frac{\kappa_{R}}{n}\right) \mathrm{d} s\right] \\
& \leq \psi_{n}\left(\left|\Delta_{0}\right|\right)+K \frac{\kappa_{R}}{n} t+\int_{0}^{t} \kappa_{R} \rho\left(\mathbb{E}\left[\left|\Delta_{s \wedge \tau_{R} \wedge S_{\delta_{0}}}\right|\right]\right) \mathrm{d} s,
\end{aligned}
$$

where the second inequality follows from the concavity of $\rho$ and Jensen's inequality. Upon passing to the limit as $n \rightarrow \infty$, we obtain from the third equation in (24) and the monotone convergence theorem that

$$
\mathbb{E}\left[\left|\Delta_{t \wedge S_{\delta_{0}} \wedge \tau_{R}}\right|\right] \leq\left|\Delta_{0}\right|+\kappa_{R} \int_{0}^{t} \rho\left(\mathbb{E}\left[\left|\Delta_{s \wedge \tau_{R} \wedge S_{\delta_{0}}}\right|\right]\right) \mathrm{d} s .
$$

When $\Delta_{0}=0$, Bihari's inequality then implies that $\mathbb{E}\left[\left|\Delta_{t \wedge \tau_{R} \wedge S_{\delta_{0}}}\right|\right]=0$. Hence by Fatou's lemma, we have $\mathbb{E}\left[\left|\Delta_{t \wedge S_{\delta_{0}}}\right|\right]=0$. This implies that $\Delta_{t \wedge S_{\delta_{0}}}=0$ a.s.

On the set $\left\{S_{\delta_{0}} \leq t\right\}$, we have $\left|\Delta_{t \wedge S_{\delta_{0}}}\right| \geq \delta_{0}$. Thus it follows that $0=\mathbb{E}\left[\left|\Delta_{t \wedge S_{\delta_{0}}}\right|\right] \geq$ $\delta_{0} \mathbb{P}\left\{S_{\delta_{0}} \leq t\right\}$. Then, we have $\mathbb{P}\left\{S_{\delta_{0}} \leq t\right\}=0$ and hence $\Delta_{t}=0$ a.s. The desired pathwise uniqueness for (21) then follows from the fact that $\widetilde{X}$ and $X$ have right continuous sample paths. Next similar to the proof of Theorem 5.1 of $\mathrm{Li}$ and $\mathrm{Pu}$ (2012), (21) has a weak solution, which further yields that the existence and uniqueness of a non-explosive strong solution to (21).

Theorem 2.5 Under Assumptions 2.1 and 2.2, for any $(x, k) \in \mathbb{R}^{d} \times \mathbb{S}$, the system given by (1) and (4) has a unique non-explosive strong solution $(X, \Lambda)$ with initial condition $(X(0), \Lambda(0))=(x, k)$.

Proof. The proof is divided into two steps. First, we show that (1) and (4) has a non-explosive solution. The second step then derives the pathwise uniqueness for 
(1) and (4). While the proof of the existence of a solution to (1) and (4) use the same line of arguments as in the proof of Theorem 2.1 of Xi and Zhu (2017), some care are required here since the assumptions in Xi and Zhu (2017) have been relaxed. Moreover, an error in the proof of Xi and Zhu (2017) is corrected here. The proof for pathwise uniqueness is more delicate than that in Xi and Zhu (2017) since the global Lipschitz conditions with respect to the variable $x$ in Xi and Zhu (2017) are no longer true in this paper.

Step 1. Let $\left(\Omega, \mathscr{F},\left\{\mathscr{F}_{t}\right\}_{t \geq 0}, \mathbb{P}\right)$ be a complete filtered probability space, on which are defined a $d$-dimensional standard Brownian motion $B$, and a Poisson random measure $N(\cdot, \cdot)$ on $[0, \infty) \times U$ with a $\sigma$-finite characteristic measure $v$ on $U$. In addition, let $\left\{\xi_{n}\right\}$ be a sequence of independent exponential random variables with mean 1 on $\left(\Omega, \mathscr{F},\left\{\mathscr{F}_{t}\right\}_{t \geq 0}, \mathbb{P}\right)$ that is independent of $B$ and $N$.

Let $k \in \mathbb{S}$ and consider the stochastic differential equation

$$
\begin{array}{r}
X^{(k)}(t)=x+\int_{0}^{t} b\left(X^{(k)}(s), k\right) \mathrm{d} s+\int_{0}^{t} \sigma\left(X^{(k)}(s), k\right) \mathrm{d} W(s) \\
+\int_{0}^{t} \int_{U} c\left(X^{(k)}(s-), k, u\right) \widetilde{N}(\mathrm{~d} s, \mathrm{~d} u) .
\end{array}
$$

Lemma 2.4 guarantees that SDE (27) has a unique non-explosive strong solution $X^{(k)}$. As in the proof of Theorem 2.1 of Xi and Zhu (2017), we define

$$
\tau_{1}=\theta_{1}:=\inf \left\{t \geq 0: \int_{0}^{t} q_{k}\left(X^{(k)}(s)\right) \mathrm{d} s>\xi_{1}\right\} .
$$

Thanks to $(10)$, we have $\mathbb{P}\left(\tau_{1}>0\right)=1$. We define a process $(X, \Lambda)$ on $\left[0, \tau_{1}\right]$ as

$$
X(t)=X^{(k)}(t) \text { for all } t \in\left[0, \tau_{1}\right] \text {, and } \Lambda(t)=k \text { for all } t \in\left[0, \tau_{1}\right) .
$$

Moreover, we define $\Lambda\left(\tau_{1}\right) \in \mathbb{S}$ according to the probability distribution

$$
\mathbb{P}\left\{\Lambda\left(\tau_{1}\right)=l \mid \mathscr{F}_{\tau_{1}-}\right\}=\frac{q_{k l}\left(X\left(\tau_{1}-\right)\right)}{q_{k}\left(X\left(\tau_{1}-\right)\right)}\left(1-\delta_{k l}\right) \mathbf{1}_{\left\{q_{k}\left(X\left(\tau_{1}-\right)\right)>0\right\}}+\delta_{k l} \mathbf{1}_{\left\{q_{k}\left(X\left(\tau_{1}-\right)\right)=0\right\}},
$$

for $l \in \mathbb{S}$. In general, having determined $(X, \Lambda)$ on $\left[0, \tau_{n}\right]$, we let

$$
\theta_{n+1}:=\inf \left\{t \geq 0: \int_{0}^{t} q_{\Lambda\left(\tau_{n}\right)}\left(X^{\left(\Lambda\left(\tau_{n}\right)\right)}(s)\right) \mathrm{d} s>\xi_{n+1}\right\}
$$

where

$$
\begin{aligned}
X^{\left(\Lambda\left(\tau_{n}\right)\right)}(t):= & X\left(\tau_{n}\right)+\int_{0}^{t} \sigma\left(X^{\left(\Lambda\left(\tau_{n}\right)\right)}(s), \Lambda\left(\tau_{n}\right)\right) \mathrm{d} B(s)+\int_{0}^{t} b\left(X^{\left(\Lambda\left(\tau_{n}\right)\right)}(s), \Lambda\left(\tau_{n}\right)\right) \mathrm{d} s \\
& +\int_{0}^{t} \int_{U} c\left(X^{\left(\Lambda\left(\tau_{n}\right)\right)}(s-), \Lambda\left(\tau_{n}\right), u\right) \widetilde{N}(\mathrm{~d} s, \mathrm{~d} u)
\end{aligned}
$$

As before, (10) implies that $\mathbb{P}\left\{\theta_{n+1}>0\right\}=1$. Then we let 


$$
\tau_{n+1}:=\tau_{n}+\theta_{n+1}
$$

and define $(X, \Lambda)$ on $\left[\tau_{n}, \tau_{n+1}\right]$ by

$$
X(t)=X^{\left(\Lambda\left(\tau_{n}\right)\right)}\left(t-\tau_{n}\right) \text { for } t \in\left[\tau_{n}, \tau_{n+1}\right], \Lambda(t)=\Lambda\left(\tau_{n}\right) \text { for } t \in\left[\tau_{n}, \tau_{n+1}\right),
$$

and

$$
\begin{aligned}
\mathbb{P}\left\{\Lambda\left(\tau_{n+1}\right)=l \mid \mathscr{F}_{\tau_{n+1}-}\right\} & =\delta_{\Lambda\left(\tau_{n}\right), l} \mathbf{1}_{\left\{q_{\Lambda\left(\tau_{n}\right)}\left(X\left(\tau_{n+1}-\right)\right)=0\right\}} \\
& +\frac{q_{\Lambda\left(\tau_{n}\right), l}\left(X\left(\tau_{n+1}-\right)\right)}{q_{\Lambda\left(\tau_{n}\right)}\left(X\left(\tau_{n+1}-\right)\right)}\left(1-\delta_{\Lambda\left(\tau_{n}\right), l}\right) \mathbf{1}_{\left\{q_{\Lambda\left(\tau_{n}\right)}\left(X\left(\tau_{n+1}-\right)\right)>0\right\}}
\end{aligned}
$$

As argued in Xi and Zhu (2017), this "interlacing procedure" uniquely determines a solution $(X, \Lambda) \in \mathbb{R}^{d} \times \mathbb{S}$ to (1) and (4) for all $t \in\left[0, \tau_{\infty}\right)$, where

$$
\tau_{\infty}=\lim _{n \rightarrow \infty} \tau_{n}
$$

Since the sequence $\tau_{n}$ is strictly increasing, the limit $\tau_{\infty} \leq \infty$ exists.

Next we show that $\tau_{\infty}=\infty$ a.s. To this end, fix $(X(0), \Lambda(0))=(x, k) \in \mathbb{R}^{d} \times \mathbb{S}$ as in Step 1 and for any $m \geq k+1$, denote by $\widetilde{\tau}_{m}:=\inf \{t \geq 0: \Lambda(t) \geq m\}$ the first exit time for the $\Lambda$ component from the finite set $\{0,1, \ldots, m-1\}$. Let $A^{c}:=\{\omega \in \Omega$ : $\tau_{\infty}>\widetilde{\tau}_{m}$ for all $\left.m \geq k+1\right\}$ and $A:=\left\{\omega \in \Omega: \tau_{\infty} \leq \widetilde{\tau}_{m_{0}}\right.$ for some $\left.m_{0} \geq k+1\right\}$. Then we have

$$
\mathbb{P}\left\{\tau_{\infty}=\infty\right\}=\mathbb{P}\left\{\tau_{\infty}=\infty \mid A^{c}\right\} \mathbb{P}\left(A^{c}\right)+\mathbb{P}\left\{\tau_{\infty}=\infty \mid A\right\} \mathbb{P}(A)
$$

Let $A_{m}:=\left\{\omega \in \Omega: \tau_{\infty} \leq \widetilde{\tau}_{m}\right\}$ for $m \geq k+1$. Then $A=\bigcup_{m=k+1}^{\infty} A_{m}$, and $A^{c}=$ $\bigcap_{m=k+1}^{\infty} A_{m}^{c}$. Also denote $B_{k+1}:=A_{k+1}$ and let

$$
B_{m}:=A_{m} \backslash A_{m-1}=\left\{\omega \in \Omega: \widetilde{\tau}_{m-1}<\tau_{\infty} \leq \widetilde{\tau}_{m}\right\}
$$

for $m \geq k+2$. Clearly, $\left\{B_{m}\right\}_{m=k+1}^{\infty}$ is a sequence of disjoint sets and we have

$$
A:=\bigcup_{m=k+1}^{\infty} B_{m} .
$$

We proceed to show that $\mathbb{P}\left\{\tau_{\infty}=\infty \mid B_{m}\right\}=1$ for each $m$. Note that on the set $B_{m}, \Lambda\left(\tau_{n}\right) \leq m$ for all $n=1,2, \ldots$ Consequently, using (10) in Assumption 2.1, we have $q_{\Lambda\left(\tau_{n}\right)}\left(X^{\left(\Lambda\left(\tau_{n}\right)\right)}(s)\right) \leq H m$ for all $n$ and $s \geq 0$. On the other hand, thanks to the definition of $\theta_{1}$ in (28), for any $\varepsilon>0$, we have

$$
\xi_{1}<\int_{0}^{\theta_{1}+\varepsilon} q_{k}\left(X^{(k)}(s)\right) \mathrm{d} s .
$$

Consequently, it follows that 


$$
\mathbf{1}_{B_{m}} \xi_{1} \leq \mathbf{1}_{B_{m}} \int_{0}^{\theta_{1}+\varepsilon} q_{k}\left(X^{(k)}(s)\right) \mathrm{d} s \leq \mathbf{1}_{B_{m}} H m\left(\theta_{1}+\varepsilon\right) .
$$

In the same manner, we have from (30) that

$$
\xi_{n}<\int_{0}^{\theta_{n}+\varepsilon / 2^{n}} q_{\Lambda\left(\tau_{n-1}\right)}\left(X^{\left(\Lambda\left(\tau_{n-1}\right)\right)}(s)\right) \mathrm{d} s,
$$

and hence

$\mathbf{1}_{B_{m}} \xi_{n} \leq \mathbf{1}_{B_{m}} \int_{0}^{\theta_{n}+\varepsilon / 2^{n}} q_{\Lambda\left(\tau_{n-1}\right)}\left(X^{\left(\Lambda\left(\tau_{n-1}\right)\right)}(s)\right) \mathrm{d} s \leq \mathbf{1}_{B_{m}} H m\left(\theta_{n}+\varepsilon / 2^{n}\right), \quad \forall n=1,2, \ldots$

Summing over these inequalities and noting that $\tau_{\infty}=\sum_{n=1}^{\infty} \theta_{n}$, we arrive at

$$
\mathbf{1}_{B_{m}} \sum_{n=1}^{\infty} \xi_{n} \leq \mathbf{1}_{B_{m}} \operatorname{Hm}\left(\tau_{\infty}+2 \varepsilon\right)
$$

By virtue of Theorem 2.3.2 of Norris (1998), we have $\sum_{n=1}^{\infty} \xi_{n}=\infty$ a.s. Therefore it follows that $\mathbb{P}\left(\sum_{n=1}^{\infty} \xi_{n}=\infty \mid B_{m}\right)=1$. Then (37) implies that

$$
\mathbb{P}\left\{\tau_{\infty}=\infty \mid B_{m}\right\} \geq \mathbb{P}\left\{\sum_{n=1}^{\infty} \xi_{n}=\infty \mid B_{m}\right\}=1,
$$

as desired. Consequently, we can use (36) to compute

$$
\begin{aligned}
\mathbb{P}\left\{\tau_{\infty}=\infty \mid A\right\} & =\frac{\mathbb{P}\left\{\tau_{\infty}=\infty, A\right\}}{\mathbb{P}(A)}=\frac{\mathbb{P}\left\{\tau_{\infty}=\infty, \bigcup_{m=k+1}^{\infty} B_{m}\right\}}{\mathbb{P}(A)} \\
& =\frac{\sum_{m=k+1}^{\infty} \mathbb{P}\left\{\tau_{\infty}=\infty, B_{m}\right\}}{\mathbb{P}(A)}=\frac{\sum_{m=k+1}^{\infty} \mathbb{P}\left\{\tau_{\infty}=\infty \mid B_{m}\right\} \mathbb{P}\left(B_{m}\right)}{\mathbb{P}(A)} \\
& =\frac{\sum_{m=k+1}^{\infty} \mathbb{P}\left(B_{m}\right)}{\mathbb{P}(A)}=1 .
\end{aligned}
$$

If $\mathbb{P}(A)=1$ or $\mathbb{P}\left(A^{c}\right)=0$, then (35) and (38) imply that $\mathbb{P}\left\{\tau_{\infty}=\infty\right\}=1$ and the proof is complete. Therefore, it remains to consider the case when $\mathbb{P}\left(A^{c}\right)>0$. Denote $\widetilde{\tau}_{\infty}:=\lim _{m \rightarrow \infty} \widetilde{\tau}_{m}$. Note that $A^{c}=\left\{\tau_{\infty} \geq \widetilde{\tau}_{\infty}\right\}$. Thus $\mathbb{P}\left\{\tau_{\infty}=\infty \mid A^{c}\right\} \geq \mathbb{P}\left\{\widetilde{\tau}_{\infty}=\right.$ $\left.\infty \mid A^{c}\right\}$ and hence (35) holds if we can show that

$$
\mathbb{P}\left\{\tilde{\tau}_{\infty}=\infty \mid A^{c}\right\}=1 .
$$

Assume on the contrary that (39) were false, then there would exist a $T>0$ such that

$$
\delta:=\mathbb{P}\left\{\tilde{\tau}_{\infty} \leq T, A^{c}\right\}>0 .
$$

Let $f: \mathbb{S} \mapsto \mathbb{R}_{+}$be as in Assumption 2.1. Then we have for any $m \geq k+1$,

$$
f(k)=\mathbb{E}\left[e^{-H\left(T \wedge \tau_{\infty} \wedge \widetilde{\tau}_{m}\right)} f\left(\Lambda\left(T \wedge \tau_{\infty} \wedge \widetilde{\tau}_{m}\right)\right)\right]
$$




$$
\begin{aligned}
& +\mathbb{E}\left[\int_{0}^{T \wedge \tau_{\infty} \wedge \widetilde{\tau}_{m}} e^{-H s}\left(H f(\Lambda(s))-\sum_{l \in \mathbb{S}} q_{\Lambda(s), l}(X(s))[f(l)-f(\Lambda(s))]\right) \mathrm{d} s\right] \\
\geq & \mathbb{E}\left[e^{-H\left(T \wedge \tau_{\infty} \wedge \widetilde{\tau}_{m}\right)} f\left(\Lambda\left(T \wedge \tau_{\infty} \wedge \widetilde{\tau}_{m}\right)\right)\right] \\
& +\mathbb{E}\left[\int_{0}^{T \wedge \tau_{\infty} \wedge \widetilde{\tau}_{m}} e^{-H s}[H f(\Lambda(s))-H(1+\Phi(X(s))+f(\Lambda(s)))] \mathrm{d} s\right] \\
\geq & \mathbb{E}\left[e^{-H\left(T \wedge \tau_{\infty} \wedge \widetilde{\tau}_{m}\right)} f\left(\Lambda\left(T \wedge \tau_{\infty} \wedge \widetilde{\tau}_{m}\right)\right)\right],
\end{aligned}
$$

where the first inequality above follows from (11) in Assumption 2.1. Consequently, we have

$$
\begin{aligned}
e^{H T} f(k) & \geq \mathbb{E}\left[f\left(\Lambda\left(T \wedge \tau_{\infty} \wedge \widetilde{\tau}_{m}\right)\right)\right] \geq \mathbb{E}\left[f\left(\Lambda\left(\widetilde{\tau}_{m}\right)\right) \mathbf{1}_{\left\{\widetilde{\tau}_{m} \leq T \wedge \tau_{\infty}\right\}}\right] \\
& \geq f(m) \mathbb{P}\left\{\widetilde{\tau}_{m} \leq T \wedge \tau_{\infty}\right\} \geq f(m) \mathbb{P}\left\{\widetilde{\tau}_{m} \leq T \wedge \tau_{\infty}, A^{c}\right\} \\
& \geq f(m) \mathbb{P}\left\{\widetilde{\tau}_{\infty} \leq T \wedge \tau_{\infty}, A^{c}\right\}
\end{aligned}
$$

where the third inequality follows from the facts that $\Lambda\left(\widetilde{\tau}_{m}\right) \geq m$ and that $f$ is nondecreasing, and the last inequality follows from the fact that $\widetilde{\tau}_{m} \uparrow \widetilde{\tau}_{\infty}$. Recall that $A^{c}=\left\{\tau_{\infty} \geq \widetilde{\tau}_{\infty}\right\}$. Thus

$$
\begin{aligned}
\mathbb{P}\left\{\widetilde{\tau}_{\infty} \leq T \wedge \tau_{\infty}, A^{c}\right\} & =\mathbb{P}\left\{\tilde{\tau}_{\infty} \leq T \wedge \tau_{\infty}, \tilde{\tau}_{\infty} \leq \tau_{\infty}\right\} \\
& \geq \mathbb{P}\left\{\widetilde{\tau}_{\infty} \leq T, \widetilde{\tau}_{\infty} \leq \tau_{\infty}\right\}=\mathbb{P}\left\{\widetilde{\tau}_{\infty} \leq T, A^{c}\right\}=\delta>0
\end{aligned}
$$

Using this observation in (40) yields $\infty>e^{H T} f(k) \geq f(m) \delta \rightarrow \infty$ as $m \rightarrow \infty$, thanks to the fact that $f(m) \rightarrow \infty$ as $m \rightarrow \infty$, which is a contradiction. This establishes (39) and hence $\mathbb{P}\left(\tau_{\infty}=\infty\right)=1$. In other words, the interlacing procedure uniquely determines a solution $(X, \Lambda)=\left(X^{(x, k)}, \Lambda^{(x, k)}\right)$ for all $t \in[0, \infty)$.

Next we show that the solution $(X, \Lambda)$ to the system (1) and (4) is non-explosive a.s. Consider the function $V(x, k):=1+\Phi(x)+f(k)$, where the functions $\Phi: \mathbb{R}^{d} \mapsto$ $\mathbb{R}_{+}$of (12) and $f: \mathbb{S} \mapsto \mathbb{R}_{+}$are defined in Assumption 2.1. Note that $V(x, k) \rightarrow \infty$ as $|x| \vee k \rightarrow \infty$ thanks to Assumption 2.1. Using the definition of $\mathscr{A}$ of (5), we have

$$
\mathscr{A} V(x, k)=\mathscr{L}_{k} \Phi(x)+Q(x) f(k) .
$$

Moreover, detailed computations using (8) and (9) reveal that $\mathscr{L}_{k} \Phi(x) \leq H \Phi(x)$ for all $x \in \mathbb{R}^{d}$ and $k \in \mathbb{S}$. On the other hand, (11) implies that $Q(x) f(k) \leq H(1+\Phi(x)+$ $f(k))$. Combining these estimates, we obtain $\mathscr{A} V(x, k) \leq 2 H V(x, k)$. This, together with Itô's formula, shows that the process $\left\{e^{-2 H t} V(X(t), \Lambda(t)), t \geq 0\right\}$ is a nonnegative local supermartingale. Then we can apply the optional sampling theorem to the process $\left\{e^{-2 H t} V(X(t), \Lambda(t)), t \geq 0\right\}$ to argue that $\mathbb{P}\left\{\lim _{n \rightarrow \infty} T_{n}=\infty\right\}=1$, where $T_{n}:=\inf \{t \geq 0:|X(t)| \vee \Lambda(t) \geq n\}$. This shows that the solution $(X, \Lambda)$ has no finite explosion time a.s.

Step 2. Suppose $(X, \Lambda)$ and $(\widetilde{X}, \widetilde{\Lambda})$ are two solutions to (1) and (4) starting from the same initial condition $(x, k) \in \mathbb{R}^{d} \times \mathbb{S}$. Then we have 


$$
\begin{aligned}
\widetilde{X}(t)-X(t)= & \int_{0}^{t}[b(\widetilde{X}(s), \widetilde{\Lambda}(s))-b(X(s), \Lambda(s))] \mathrm{d} s \\
& +\int_{0}^{t}[\sigma(\widetilde{X}(s), \widetilde{\Lambda}(s))-\sigma(X(s), \Lambda(s))] \mathrm{d} W(s) \\
& +\int_{U}[c(\widetilde{X}(s-), \widetilde{\Lambda}(s-), z)-c(X(s-), \Lambda(s-), z)] \widetilde{N}(\mathrm{~d} s, \mathrm{~d} u)
\end{aligned}
$$

and

$$
\widetilde{\Lambda}(t)-\Lambda(t)=\int_{0}^{t} \int_{\mathbb{R}_{+}}[h(\widetilde{X}(s-), \widetilde{\Lambda}(s-), z)-h(X(s-), \Lambda(s-), z)] N_{1}(\mathrm{~d} s, \mathrm{~d} z) .
$$

Let $\zeta:=\inf \{t \geq 0: \Lambda(t) \neq \widetilde{\Lambda}(t)\}$ be the first time when the discrete components differ from each other. Let us also define $T_{R}:=\inf \{t \geq 0:|\widetilde{X}(t)| \vee|X(t)| \vee \widetilde{\Lambda}(t) \vee$ $\Lambda(t) \geq R\}$ for $R>0$ and $S_{\delta_{0}}:=\inf \left\{t \geq 0:|\widetilde{X}(t)-X(t)| \geq \delta_{0}\right\}$. We have $\Lambda(t)=\widetilde{\Lambda}(t)$ for $t \in[0, \zeta)$. To simplify notation, let us define $\Delta_{t}:=\widetilde{X}(t)-X(t)$. Then from the proof of Theorem 2.6 of Xi and Zhu (2018a), we have $\mathbb{E}\left[H\left(\left|\Delta_{t \wedge \zeta \wedge S_{\delta_{0}}}\right|\right)\right]=0$ for $d \geq 2$, where $H(r):=\frac{r^{2}}{1+r^{2}}, r \geq 0$. When $d=1$, the proof of Lemma 2.4 reveals that $\mathbb{E}\left[\left|\Delta_{t \wedge \zeta \wedge S_{\delta_{0}}}\right|\right]=0$ and hence $\mathbb{E}\left[H\left(\left|\Delta_{t \wedge \zeta \wedge S_{\delta_{0}}}\right|\right)\right]=0$. Note that on the set $\left\{S_{\delta_{0}} \leq t \wedge \zeta\right\}$, $\left|\Delta_{t \wedge \zeta \wedge S_{\delta_{0}}}\right| \geq \delta_{0}$. Also we can readily check that $H$ is an increasing function. Thus, it follows that

$$
0=\mathbb{E}\left[H\left(\left|\Delta_{t \wedge \zeta \wedge S_{\delta_{0}}}\right|\right)\right] \geq \mathbb{E}\left[H\left(\left|\Delta_{t \wedge \zeta \wedge S_{\delta_{0}}}\right|\right) \mathbf{1}_{\left\{S_{\delta_{0}} \leq t \wedge \zeta\right\}}\right] \geq H\left(\delta_{0}\right) \mathbb{P}\left\{S_{\delta_{0}} \leq t \wedge \zeta\right\} .
$$

This implies that $\mathbb{P}\left\{S_{\delta_{0}} \leq t \wedge \zeta\right\}=0$. Consequently, we have

$$
\begin{aligned}
\mathbb{E}\left[H\left(\left|\Delta_{t \wedge \zeta}\right|\right)\right] & =\mathbb{E}\left[H\left(\left|\Delta_{t \wedge \zeta}\right|\right) \mathbf{1}_{\left\{S_{\delta_{0}} \leq t \wedge \zeta\right\}}\right]+\mathbb{E}\left[H\left(\left|\Delta_{t \wedge \zeta}\right|\right) \mathbf{1}_{\left\{S_{\delta_{0}}>t \wedge \zeta\right\}}\right] \\
& \leq \mathbb{P}\left\{S_{\delta_{0}} \leq t \wedge \zeta\right\}+\mathbb{E}\left[H\left(\left|\Delta_{t \wedge \zeta \wedge S_{\delta_{0}}}\right|\right) \mathbf{1}_{\left\{S_{\delta_{0}}>t \wedge \zeta\right\}}\right] \\
& \leq 0 .
\end{aligned}
$$

It follows that $\mathbb{E}\left[\left|\Delta_{t \wedge \zeta}\right|\right]=\mathbb{E}[|\widetilde{X}(t \wedge \zeta)-X(t \wedge \zeta)|]=0$. Then we have

$$
\mathbb{E}\left[|\widetilde{X}(t \wedge \zeta)-X(t \wedge \zeta)|^{\delta}\right]=0,
$$

where $\delta \in(0,1]$ is the Hölder constant in (13).

Note that $\zeta \leq t$ if and only if $\widetilde{\Lambda}(t \wedge \zeta)-\Lambda(t \wedge \zeta) \neq 0$. Therefore, it follows that

$$
\begin{array}{r}
\mathbb{P}\{\zeta \leq t\}=\mathbb{E}\left[\mathbf{1}_{\{\tilde{\Lambda}(t \wedge \zeta)-\Lambda(t \wedge \zeta) \neq 0\}}\right] \\
=\mathbb{E}\left[\int _ { 0 } ^ { t \wedge \zeta } \int _ { \mathbb { R } _ { + } } \left(\mathbf{1}_{\{\widetilde{\Lambda}(s-)-\Lambda(s-)+h(\widetilde{X}(s-), \Lambda(s-), z)-h(X(s-), \Lambda(s-), z) \neq 0\}}\right.\right. \\
\left.\left.\quad-\mathbf{1}_{\{\widetilde{\Lambda}(s-)-\Lambda(s-) \neq 0\}}\right) \mathfrak{m}(\mathrm{d} z) \mathrm{d} s\right]
\end{array}
$$




$$
\begin{aligned}
& =\mathbb{E}\left[\int_{0}^{t \wedge \zeta} \int_{\mathbb{R}_{+}} \mathbf{1}_{\{h(\widetilde{X}(s-), \Lambda(s-), z)-h(X(s-), \Lambda(s-), z) \neq 0\}} \mathfrak{m}(\mathrm{d} z) \mathrm{d} s\right] \\
& \leq \mathbb{E}\left[\int_{0}^{t \wedge \zeta} \sum_{l \in \mathbb{S}, l \neq \Lambda(s-)}\left|q_{\Lambda(s-), l}(\widetilde{X}(s-))-q_{\Lambda(s-), l}(X(s-))\right| \mathrm{d} s\right] \\
& \left.\leq H \mathbb{E}\left[\int_{0}^{\wedge \zeta \zeta} \mid \widetilde{X}(s-)\right)-\left.X(s-)\right|^{\delta} \mathrm{d} s\right] \leq H \int_{0}^{t} \mathbb{E}\left[|\widetilde{X}(s \wedge \zeta)-X(s \wedge \zeta)|^{\delta}\right] \mathrm{d} s=0,
\end{aligned}
$$

where the second inequality follows from (13). In particular, we have

$$
\mathbb{E}\left[\mathbf{1}_{\{\tilde{\Lambda}(t) \neq \Lambda(t)\}}\right] \leq \mathbb{P}\{\zeta \leq t\}=0 .
$$

Now we can compute

$$
\begin{aligned}
\mathbb{E}[H(|\widetilde{X}(t)-X(t)|)] & =\mathbb{E}\left[H(|\widetilde{X}(t)-X(t)|) \mathbf{1}_{\{\zeta>t\}}\right]+\mathbb{E}\left[H(|\widetilde{X}(t)-X(t)|) \mathbf{1}_{\{\zeta \leq t\}}\right] \\
& =\mathbb{E}\left[H(|\widetilde{X}(t \wedge \zeta)-X(t \wedge \zeta)|) \mathbf{1}_{\{\zeta>t\}}\right]+\mathbb{E}\left[1 \cdot \mathbf{1}_{\{\zeta \leq t\}}\right] \\
& \leq \mathbb{E}[H(|\widetilde{X}(t \wedge \zeta)-X(t \wedge \zeta)|)]+0 \\
& =0 .
\end{aligned}
$$

Thus $\mathbb{P}\{\tilde{X}(t)=X(t)\}=1$. This, together with (42), implies that $\mathbb{P}\{(\widetilde{X}(t), \widetilde{\Lambda}(t))=$ $(X(t), \Lambda(t))\}=1$ for all $t \geq 0$. Since the sample paths of $(X, \Lambda)$ are right continuous, we obtain the desired pathwise uniqueness result.

Example 2.6 Let us consider the following SDE

$$
\begin{aligned}
\mathrm{d} X(t)= & b(X(t), \Lambda(t)) \mathrm{d} t+\sigma(X(t), \Lambda(t)) \mathrm{d} W(t) \\
& +\int_{U} c(X(t-), \Lambda(t-), u) \widetilde{N}(\mathrm{~d} t, \mathrm{~d} u), \quad X(0)=x \in \mathbb{R}^{3},
\end{aligned}
$$

where $W$ is a 3-dimensional standard Brownian motion, $\widetilde{N}(\mathrm{~d} t, \mathrm{~d} u)$ is a compensated Poisson random measure with compensator $\mathrm{d} t v(\mathrm{~d} u)$ on $[0, \infty) \times U$, in which $U=\left\{u \in \mathbb{R}^{3}: 0<|u|<1\right\}$ and $v(\mathrm{~d} u):=\frac{\mathrm{d} u}{|u|^{3+\alpha}}$ for some $\alpha \in(0,2)$. The $\Lambda$ component in (43) takes value in $\mathbb{S}=\{1,2, \ldots\}$ and is generated by $Q(x)=\left(q_{k l}(x)\right)$, with $q_{k l}(x)=\frac{k}{2^{l}} \cdot \frac{|x|^{2}}{1+|x|^{2}}$ for $x \in \mathbb{R}^{3}$ and $k \neq l \in \mathbb{S}$. Let $q_{k}(x)=-q_{k k}(x)=\sum_{l \neq k} q_{k l}(x)$. The coefficients of (43) are given by

$$
b(x, k)=\left(\begin{array}{c}
-x_{1}^{1 / 3}-k x_{1}^{3} \\
-x_{2}^{1 / 3}-k x_{2}^{3} \\
-x_{3}^{1 / 3}-k x_{3}^{3}
\end{array}\right), \quad c(x, k, u)=c(x, u)=\left(\begin{array}{c}
\gamma x_{1}^{2 / 3}|u| \\
\gamma x_{2}^{2 / 3}|u| \\
\gamma x_{3}^{2 / 3}|u|
\end{array}\right)
$$

and 


$$
\sigma(x, k)=\left(\begin{array}{ccc}
\frac{x_{1}^{2 / 3}}{\sqrt{2}}+1 & \frac{\sqrt{k} x_{2}^{2}}{3} & \frac{\sqrt{k} x_{3}^{2}}{3} \\
\frac{\sqrt{k} x_{1}^{2}}{3} & \frac{x_{2}^{2 / 3}}{\sqrt{2}}+1 & \frac{\sqrt{k} x_{3}^{2}}{3} \\
\frac{\sqrt{k} x_{1}^{2}}{3} & \frac{\sqrt{k} x_{2}^{2}}{3} & \frac{x_{3}^{2 / 3}}{\sqrt{2}}+1
\end{array}\right),
$$

in which $\gamma$ is a positive constant so that $\gamma^{2} \int_{U}|u|^{2} v(\mathrm{~d} u)=\frac{1}{2}$.

Note that $\sigma$ and $b$ grow very fast in the neighborhood of $\infty$ and they are Hölder continuous with orders $\frac{2}{3}$ and $\frac{1}{3}$, respectively. Nevertheless, the coefficients of (43) still satisfy Assumptions 2.2 and 2.1 and hence a unique non-exploding strong solution of (43) exists. The verifications of these assumptions are as follows.

$$
\begin{aligned}
& 2\langle x, b(x, k)\rangle+|\sigma(x, k)|^{2}+\int_{U}|c(x, k, u)|^{2} v(\mathrm{~d} u) \\
& =2 \sum_{j=1}^{3} x_{j}\left(-x_{j}^{1 / 3}-k x_{j}^{3}\right)+\sum_{j=1}^{3}\left(\frac{1}{2} x_{j}^{4 / 3}+\frac{2 k}{9} x_{j}^{4}+\sqrt{2} x_{j}^{2 / 3}+1\right) \\
& \quad+\int_{U} \gamma^{2}|u|^{2} \sum_{j=1}^{3} x_{j}^{4 / 3} v(\mathrm{~d} u) \\
& =-\frac{16 k}{9} \sum_{j=1}^{3} x_{j}^{4}-\sum_{j=1}^{3} x_{j}^{4 / 3}+\sqrt{2} \sum_{j=1}^{3} x_{j}^{2 / 3}+3 .
\end{aligned}
$$

Thus (9) of Assumption 2.1 hold. Furthermore, (10) is trivially satisfied. Consider the function $f(l)=l, l \in \mathbb{S}$. We have

$$
\sum_{l \neq k}(f(l)-f(k)) q_{k l}(x)=\sum_{l \neq k}(l-k) \frac{k}{2^{l}} \frac{|x|^{2}}{1+|x|^{2}} \leq \sum_{l \neq k} l \frac{k}{2^{l}} \frac{|x|^{2}}{1+|x|^{2}} \leq k \sum_{l \in \mathbb{S}} \frac{l}{2^{l}}=2 k,
$$

which yields (11). If $x, y \in \mathbb{R}^{3}$, we obtain

$$
\begin{aligned}
\sum_{l \neq k}\left|q_{k l}(x)-q_{k l}(y)\right| & =\sum_{l \neq k} \frac{l}{2^{k}}\left|\frac{|x|^{2}}{1+|y|^{2}}-\frac{|x|^{2}}{1+|y|^{2}}\right|=\sum_{l \neq k} \frac{l}{2^{k}} \frac{|| x|-| y||(|x|+|y|)}{\left(1+|x|^{2}\right)\left(1+|y|^{2}\right)} \\
& \leq \sum_{l \neq k} \frac{l}{2^{k}}|x-y|\left(\frac{|x|}{1+|x|^{2}}+\frac{|y|}{1+|y|^{2}}\right) \leq 2|x-y| .
\end{aligned}
$$

This establishes (13) and therefore verifies Assumption 2.1.

For the verification of Assumption 2.2, we compute

$$
\begin{aligned}
& 2\langle x-y, b(x, k)-b(y, k)\rangle+|\sigma(x, k)-\sigma(y, k)|^{2}+\int_{U}|c(x, k, u)-c(y, k, u)|^{2} v(\mathrm{~d} u) \\
& =-2 \sum_{j=1}^{3}\left(x_{j}-y_{j}\right)\left(x_{j}^{1 / 3}-y_{j}^{1 / 3}+k x_{j}^{3}-k y_{j}^{3}\right)+\frac{1}{2} \sum_{j=1}^{3}\left(x_{j}^{2 / 3}-y_{j}^{2 / 3}\right)^{2}
\end{aligned}
$$




$$
\begin{gathered}
+\frac{2 k}{9} \sum_{j=1}^{3}\left(x_{j}^{2}-y_{j}^{2}\right)^{2}+\int_{U} \sum_{j=1}^{3} \gamma^{2}\left(x_{j}^{2 / 3}-y_{j}^{2 / 3}\right)^{2}|u|^{2} v(\mathrm{~d} u) \\
=-\frac{16 k}{9} \sum_{j=1}^{3}\left(x_{j}-y_{j}\right)^{2}\left[\left(x_{j}+\frac{7}{16} y_{j}\right)^{2}+\frac{207}{256} y_{j}^{2}\right]-\sum_{j=1}^{3}\left(x_{j}^{1 / 3}-y_{j}^{1 / 3}\right)^{2}\left(x_{j}^{2 / 3}+y_{j}^{2 / 3}\right) .
\end{gathered}
$$

Obviously this implies (20) and thus verifies Assumption 2.2.

\section{Feller Property}

In Section 2, we established the existence and uniqueness of a solution in the strong sense to system (1) and (4) under Assumptions 2.1 and 2.2. The solution $(X, \Lambda)$ is a two-component càdlàg strong Markov process. In this section, we study the Feller property for such processes. For any $f \in C_{b}\left(\mathbb{R}^{d} \times \mathbb{S}\right)$, by the continuity of $f$ and the right continuity of the sample paths of $(X, \Lambda)$, we can use the bounded convergence theorem to obtain $\lim _{t \downarrow 0} \mathbb{E}_{x, k}[f(X(t), \Lambda(t))]=f(x, k)$. Therefore the process $(X, \Lambda)$ satisfies the Feller property if the semigroup $P_{t} f(x, k):=\mathbb{E}_{x, k}[f(X(t), \Lambda(t))], f \in$ $\mathfrak{B}_{b}\left(\mathbb{R}^{d} \times \mathbb{S}\right)$ maps $C_{b}\left(\mathbb{R}^{d} \times \mathbb{S}\right)$ into itself. Obviously, to establish the Feller property, we only need the distributional properties of the process $(X, \Lambda)$. Thus in lieu of the strong formulation used in Section 2, we will assume the following "weak formulation" throughout the section.

Assumption 3.1 For any initial data $(x, k) \in \mathbb{R}^{d} \times \mathbb{S}$, the system of stochastic differential equations (1) and (4) has a non-exploding weak solution $\left(X^{(x, k)}, \Lambda^{(x, k)}\right)$ and the solution is unique in the sense of probability law.

Assumption 3.2 There exist a positive constant $\delta_{0}$ and an increasing and concave function $\rho:[0, \infty) \mapsto[0, \infty)$ satisfying (19) such that for all $R>0$, there exists a constant $\kappa_{R}>0$ such that

$$
\sum_{l \in \mathbb{S} \backslash\{k\}}\left|q_{k l}(x)-q_{k l}(z)\right| \leq \kappa_{R} \rho(F(|x-z|)), \text { for all } k \in \mathbb{S} \text { and }|x| \vee|z| \leq R
$$

where $F(r):=\frac{r}{1+r}$ for $r \geq 0$, and either (i) or (ii) below holds:

(i) $d=1$. Then (15) and (17) hold.

(ii) $d \geq 2$. Then

$$
\begin{aligned}
& \int_{U}\left[|c(x, k, u)-c(z, k, u)|^{2} \wedge(4|x-z| \cdot|c(x, k, u)-c(z, k, u)|)\right] v(\mathrm{~d} u) \\
& \quad+2\langle x-z, b(x, k)-b(z, k)\rangle+|\sigma(x, k)-\sigma(z, k)|^{2} \leq 2 \kappa_{R}|x-z| \rho(|x-z|),
\end{aligned}
$$

for all $k \in \mathbb{S}, x, z \in \mathbb{R}^{d}$ with $|x| \vee|z| \leq R$ and $|x-z| \leq \delta_{0}$.

Theorem 3.3 Under Assumptions 3.1 and 3.2, the process $(X, \Lambda)$ possesses the Feller property. 
Remark 3.4 Feller and strong Feller properties for regime-switching (jump) diffusions have been investigated in Shao (2015), Xi and Zhu (2017), Yin and Zhu (2010), among others. A standard assumption in these references is that the coefficients satisfy the Lipschitz condition. In contrast, Theorem 3.3 establishes Feller property for system (1) and (4) under local non-Lipschitz conditions. When $d=1$, the result is even more remarkable. Indeed, Feller property is derived with only very mild conditions on $b(\cdot, k), c(\cdot, k, u)$, and $Q(x)$, and with virtually no condition imposed on $\sigma(\cdot, k)$.

We will use the coupling method to prove Theorem 3.3. To this end, let us first construct a coupling operator $\widetilde{\mathscr{A}}$ for $\mathscr{A}$ : For $f(x, i, z, j) \in C_{c}^{2}\left(\mathbb{R}^{d} \times \mathbb{S} \times \mathbb{R}^{d} \times \mathbb{S}\right)$, we define

$$
\widetilde{\mathscr{A}} f(x, i, z, j):=\left[\widetilde{\Omega}_{\mathrm{d}}+\widetilde{\Omega}_{\mathrm{j}}+\widetilde{\Omega}_{\mathrm{s}}\right] f(x, i, z, j),
$$

where $\widetilde{\Omega}_{\mathrm{d}}, \widetilde{\Omega}_{\mathrm{j}}$, and $\widetilde{\Omega}_{\mathrm{s}}$ are defined as follows. For $x, z \in \mathbb{R}^{d}$ and $i, j \in \mathbb{S}$, we set $a(x, i)=\sigma(x, i) \sigma(x, i)^{\prime}$ and

$$
a(x, i, z, j)=\left(\begin{array}{cc}
a(x, i) & \sigma(x, i) \sigma(z, j)^{\prime} \\
\sigma(z, j) \sigma(x, i)^{\prime} & a(z, j)
\end{array}\right), b(x, i, z, j)=\left(\begin{array}{c}
b(x, i) \\
b(z, j)
\end{array}\right) .
$$

Then we define

$$
\begin{aligned}
\widetilde{\Omega}_{\mathrm{d}} f(x, i, z, j):= & \frac{1}{2} \operatorname{tr}\left(a(x, i, z, j) D^{2} f(x, i, z, j)\right)+\langle b(x, i, z, j), D f(x, i, z, j)\rangle, \\
\widetilde{\Omega}_{\mathrm{j}} f(x, i, z, j):= & \int_{U}[f(x+c(x, i, u), i, z+c(z, j, u), j)-f(x, i, z, j) \\
& \left.-\left\langle D_{x} f(x, i, z, j), c(x, i, u)\right\rangle-\left\langle D_{z} f(x, i, z, j), c(z, j, u)\right\rangle\right] v(\mathrm{~d} u),
\end{aligned}
$$

where $D f(x, i, z, j)=\left(D_{x} f(x, i, z, j), D_{z} f(x, i, z, j)\right)^{\prime}$ is the gradient and $D^{2} f(x, i, z, j)$ the Hessian matrix of $f$ with respect to the $x, z$ variables, and

$$
\begin{aligned}
\widetilde{\Omega}_{\mathrm{S}} f(x, i, z, j):= & \sum_{l \in \mathbb{S}}\left[q_{i l}(x)-q_{j l}(z)\right]^{+}(f(x, l, z, j)-f(x, i, z, j)) \\
& +\sum_{l \in \mathbb{S}}\left[q_{j l}(z)-q_{i l}(x)\right]^{+}(f(x, i, z, l)-f(x, i, z, j)) \\
& +\sum_{l \in \mathbb{S}}\left[q_{i l}(x) \wedge q_{j l}(z)\right](f(x, l, z, l)-f(x, i, z, j)) .
\end{aligned}
$$

For convenience of later presentation, for any function $f: \mathbb{R}^{d} \times \mathbb{R}^{d} \mapsto \mathbb{R}$, let $\tilde{f}$ : $\mathbb{R}^{d} \times \mathbb{S} \times \mathbb{R}^{d} \times \mathbb{S} \mapsto \mathbb{R}$ be defined by $\widetilde{f}(x, i, z, j):=f(x, z)$. Now we denote

$$
\widetilde{\mathscr{L}}_{k} f(x, z)=\left(\widetilde{\Omega}_{\mathrm{d}}^{(k)}+\widetilde{\Omega}_{\mathrm{j}}^{(k)}\right) f(x, z):=\left(\widetilde{\Omega}_{\mathrm{d}}+\widetilde{\Omega}_{\mathrm{j}}\right) \widetilde{f}(x, k, z, k), \forall f \in C_{c}^{2}\left(\mathbb{R}^{d} \times \mathbb{R}^{d}\right)
$$

for each $k \in \mathbb{S}$. We proceed to establish the following lemma.

Lemma 3.5 Suppose Assumption 3.2 holds. Consider the functions 


$$
g(x, k, z, l):=\mathbf{1}_{\{k \neq l\}} \text {, and } f(x, k, z, l):=F(|x-z|)+\mathbf{1}_{\{k \neq l\}},
$$

for $(x, k, z, l) \in \mathbb{R}^{d} \times \mathbb{S} \times \mathbb{R}^{d} \times \mathbb{S}$. Then we have

$$
\widetilde{\mathscr{A} g}(x, k, z, l) \leq \kappa_{R} \rho(F(|x-y|)), \text { for all } k, l \in \mathbb{S} \text { and } x, z \in \mathbb{R}^{d} \text { with }|x| \vee|z| \leq R,
$$

and

$$
\widetilde{\mathscr{A}} f(x, k, z, k) \leq 2 \kappa_{R} \rho(F(|x-z|)),
$$

for all $k \in \mathbb{S}$ and $x, z \in \mathbb{R}^{d}$ with $|x| \vee|z| \leq R$ and $0<|x-z| \leq \delta_{0}$; in which $\kappa_{R}$ is the same positive constant as in Assumption 3.2.

Proof. Consider the function $g(x, k, z, l):=\mathbf{1}_{\{k \neq l\}}$. It follows directly from the definition that $\widetilde{\mathscr{A} g}(x, k, z, l)=\widetilde{\Omega}_{\mathrm{s}} g(x, k, z, l) \leq 0$ when $k \neq l$. When $k=l$, we have from (44) that

$$
\begin{aligned}
\widetilde{\mathscr{A} g}(x, k, z, l)= & \widetilde{\Omega}_{\mathrm{s}} g(x, k, z, k) \\
= & \sum_{i \in \mathbb{S}}\left[q_{k i}(x)-q_{k i}(z)\right]^{+}\left(\mathbf{1}_{\{i \neq k\}}-\mathbf{1}_{\{k \neq k\}}\right) \\
& \quad+\sum_{i \in \mathbb{S}}\left[q_{k i}(z)-q_{k i}(x)\right]^{+}\left(\mathbf{1}_{\{i \neq k\}}-\mathbf{1}_{\{k \neq k\}}\right)+0 \\
\leq & \sum_{i \in \mathbb{S}, i \neq k}\left|q_{k i}(x)-q_{k i}(z)\right| \leq \kappa_{R} \rho(F(|x-y|)) .
\end{aligned}
$$

Hence (51) holds for all $k, l \in \mathbb{S}$ and $x, z \in \mathbb{R}^{d}$ with $|x| \vee|z| \leq R$. On the other hand, when $d \geq 2$, (45) and Lemma 4.5 of Xi and Zhu (2018a) reveals that

$$
\widetilde{\mathscr{L}}_{k} F(|x-z|)=\left(\widetilde{\Omega}_{\mathrm{d}}^{(k)}+\widetilde{\Omega}_{\mathrm{j}}^{(k)}\right) F(|x-z|) \leq \kappa_{R} \rho(F(|x-z|))
$$

and hence

$$
\widetilde{\mathscr{A}} f(x, k, z, k)=\widetilde{\mathscr{L}_{k}} F(|x-z|)+\widetilde{\Omega}_{\mathrm{s}} g(x, k, z, k) \leq 2 \kappa_{R} \rho(F(|x-z|)),
$$

for all $k \in \mathbb{S}, x, z \in \mathbb{R}^{d}$ with $|x| \vee|z| \leq R$ and $0<|x-z| \leq \delta_{0}$, where $\widetilde{\mathscr{L}_{k}}$ is the basic coupling operator for $\mathscr{L}_{k}$ of (6).

We next show that (52) holds when $d=1$. Indeed, taking advantage of the fact that $d=1$, we see that

$$
\begin{aligned}
& \widetilde{\Omega}_{\mathrm{d}}^{(k)} F(|x-z|) \\
& \quad=F^{\prime}(|x-z|) \operatorname{sgn}(x-z)(b(x, k)-b(z, k))+F^{\prime \prime}(|x-z|)(\sigma(x, k)-\sigma(z, k))^{2} .
\end{aligned}
$$

But since $F^{\prime}(r)=\frac{1}{(1+r)^{2}}$ and $F^{\prime \prime}(r)=-\frac{2}{(1+r)^{3}}<0$ for $r \geq 0$, we have from (15) that

$$
\widetilde{\Omega}_{\mathrm{d}}^{(k)} F(|x-z|) \leq \frac{1}{(1+|x-z|)^{2}} \operatorname{sgn}(x-z)(b(x, k)-b(z, k))
$$




$$
\leq \frac{\kappa_{R} \rho(|x-z|)}{(1+|x-z|)^{2}} \leq \kappa_{R} \rho(F(|x-z|)),
$$

for all $x, z \in \mathbb{R}$ with $|x| \vee|z| \leq R$ and $0<|x-z| \leq \delta_{0}$, where we used the first equation in (19) to derive the last inequality.

On the other hand, since the function $F$ is concave on $[0, \infty)$, we have $F(r)-$ $F\left(r_{0}\right) \leq F^{\prime}\left(r_{0}\right)\left(r-r_{0}\right)$ for all $r, r_{0} \in[0, \infty)$. Applying this inequality with $r_{0}=|x-z|$ and $r=|x-z+c(x, k, u)-c(z, k, u)|$ yields

$$
\begin{aligned}
& \quad F(|x-z+c(x, k, u)-c(z, k, u)|)-F(|x-z|) \\
& \quad \leq F^{\prime}(|x-z|)(|x-z+c(x, k, u)-c(z, k, u)|-|x-z|) .
\end{aligned}
$$

Furthermore, since by (17), the function $x \mapsto x+c(x, k, u)$ is increasing, it follows that for $x>z$

$$
\begin{aligned}
& F(|x-z+c(x, k, u)-c(z, k, u)|)-F(|x-z|) \\
& \quad \leq F^{\prime}(|x-z|)(x-z+c(x, k, u)-c(z, k, u)-(x-z)) \\
& \quad=F^{\prime}(|x-z|)(c(x, k, u)-c(z, k, u)) .
\end{aligned}
$$

As a result, we can compute

$$
\begin{aligned}
\widetilde{\Omega}_{\mathrm{j}}^{(k)} F(|x-z|) \\
=\int_{U}[F(|x-z+c(x, k, u)-c(z, k, u)|)-F(|x-z|) \\
\left.\quad \quad-F^{\prime}(|x-z|) \operatorname{sgn}(x-z)(c(x, k, u)-c(z, k, u))\right] v(\mathrm{~d} u) \\
\leq \int_{U}\left[F^{\prime}(|x-z|)(c(x, k, u)-c(z, k, u))-F^{\prime}(|x-z|)(c(x, k, u)-c(z, k, u))\right] v(\mathrm{~d} u) \\
=0,
\end{aligned}
$$

for all $x>z$. By symmetry, we also have $\widetilde{\Omega}_{\mathrm{j}}^{(k)} F(|x-z|) \leq 0$ for $x<z$. These observations, together with (53) and (55), imply that

$$
\widetilde{\mathscr{A}} f(x, k, z, k)=\left(\widetilde{\Omega}_{\mathrm{d}}^{(k)}+\widetilde{\Omega}_{\mathrm{j}}^{(k)}\right) F(|x-z|)+\widetilde{\Omega}_{\mathrm{s}} g(x, k, z, k) \leq 2 \kappa_{R} \rho(F(|x-z|)),
$$

for all $k \in \mathbb{S}, x, z \in \mathbb{R}$ with $|x| \vee|z| \leq R$ and $0<|x-z| \leq \delta_{0}$ This completes the proof.

Proof (Proof of Theorem 3.3). It is straightforward to verify that the function $f$ of (50) defines a bounded metric on $\mathbb{R}^{d} \times \mathbb{S}$. Let $(\widetilde{X}(\cdot), \widetilde{\Lambda}(\cdot), \widetilde{Z}(\cdot), \widetilde{\Xi}(\cdot))$ denote the coupling process corresponding to the coupling operator $\widetilde{\mathscr{A}}$ with initial condition $(x, k, z, k)$, in which $\delta_{0}>|x-z|>0$. Define $\zeta:=\inf \{t \geq 0: \widetilde{\Lambda}(t) \neq \widetilde{\Xi}(t)\}$. Note that $\mathbb{P}\{\zeta>0\}=1$. Suppose $|x-z|>\frac{1}{n_{0}}$ for some $n_{0} \in \mathbb{N}$. For $n \geq n_{0}$ and $R>|x| \vee|z|$, define

$$
T_{n}:=\inf \left\{t \geq 0:|\widetilde{X}(t)-\widetilde{Z}(t)|<\frac{1}{n}\right\}
$$




$$
\tau_{R}:=\inf \{t \geq 0:|\widetilde{X}(t)| \vee|\widetilde{Z}(t)| \vee \widetilde{\Lambda}(t) \vee \widetilde{\Xi}(t)>R\},
$$

and

$$
S_{\delta_{0}}:=\inf \left\{t \geq 0:|\widetilde{X}(t)-\widetilde{Z}(t)|>\delta_{0}\right\} .
$$

We have $\tau_{R} \rightarrow \infty$ and $T_{n} \rightarrow T$ a.s. as $R \rightarrow \infty$ and $n \rightarrow \infty$, respectively, in which $T$ denotes the first time when $\widetilde{X}(t)$ and $\widetilde{Z}(t)$ coalesce. To simplify notation, denote $\widetilde{\Delta}(s):=\widetilde{X}(s)-\widetilde{Z}(s)$. By Itô's formula and (54), we have

$$
\begin{aligned}
& \mathbb{E}\left[F\left(\left|\widetilde{\Delta}\left(t \wedge T_{n} \wedge S_{\delta_{0}} \wedge \tau_{R} \wedge \zeta\right)\right|\right)\right] \\
& \quad \leq \mathbb{E}\left[f \left(\widetilde{X}\left(t \wedge T_{n} \wedge S_{\delta_{0}} \wedge \tau_{R} \wedge \zeta\right), \widetilde{\Lambda}\left(t \wedge T_{n} \wedge S_{\delta_{0}} \wedge \tau_{R} \wedge \zeta\right),\right.\right. \\
& \left.\left.\widetilde{Z}\left(t \wedge T_{n} \wedge S_{\delta_{0}} \wedge \tau_{R} \wedge \zeta\right), \widetilde{\Xi}\left(t \wedge T_{n} \wedge S_{\delta_{0}} \wedge \tau_{R} \wedge \zeta\right)\right)\right] \\
& =F(|x-z|)+\mathbb{E}\left[\int_{0}^{t \wedge T_{n} \wedge S_{\delta_{0}} \wedge \tau_{R} \wedge \zeta} \widetilde{\mathscr{A}} f(\widetilde{X}(s), \Delta(s), \widetilde{Z}(s), \widetilde{\Xi}(s)) \mathrm{d} s\right] \\
& \quad \leq F(|x-z|)+2 \kappa_{R} \mathbb{E}\left[\int_{0}^{t \wedge T_{n} \wedge S_{\delta_{0}} \wedge \tau_{R} \wedge \zeta} \rho(F(|\widetilde{\Delta}(s)|)) \mathrm{d} s\right] .
\end{aligned}
$$

Now passing to the limit as $n \rightarrow \infty$, it follows from the bounded and monotone convergence theorems that

$$
\begin{aligned}
& \mathbb{E}\left[F\left(\left|\widetilde{\Delta}\left(t \wedge T \wedge S_{\delta_{0}} \wedge \tau_{R} \wedge \zeta\right)\right|\right)\right] \\
& \quad \leq F(|x-z|)+2 \kappa_{R} \mathbb{E}\left[\int_{0}^{t \wedge T \wedge S_{\delta_{0}} \wedge \tau_{R} \wedge \zeta} \rho(F(|\widetilde{\Delta}(s)|)) \mathrm{d} s\right] \\
& \quad \leq F(|x-z|)+2 \kappa_{R} \mathbb{E}\left[\int_{0}^{t} \rho\left(F\left(\left|\widetilde{\Delta}\left(s \wedge T \wedge S_{\delta_{0}} \wedge \tau_{R} \wedge \zeta\right)\right|\right)\right) \mathrm{d} s\right] \\
& \quad \leq F(|x-z|)+2 \kappa_{R} \int_{0}^{t} \rho\left(\mathbb{E}\left[F\left(\left|\widetilde{\Delta}\left(s \wedge T \wedge S_{\delta_{0}} \wedge \tau_{R} \wedge \zeta\right)\right|\right)\right]\right) \mathrm{d} s,
\end{aligned}
$$

where we used the concavity of $\rho$ and Jensen's inequality to obtain the last inequality. Then using Bihari's inequality, we have

$$
\mathbb{E}\left[F\left(\left|\widetilde{\Delta}\left(t \wedge T \wedge S_{\delta_{0}} \wedge \tau_{R} \wedge \zeta\right)\right|\right)\right] \leq G^{-1}\left(G \circ F(|x-z|)+2 \kappa_{R} t\right),
$$

where the function $G(r):=\int_{1}^{r} \frac{\mathrm{d} s}{\rho(s)}$ is strictly increasing and satisfies $G(r) \rightarrow-\infty$ as $r \downarrow 0$. In addition, since the function $F$ is strictly increasing, we have

$$
\begin{aligned}
F\left(\delta_{0}\right) \mathbb{P}\left\{S_{\delta_{0}}<t \wedge T \wedge \tau_{R} \wedge \zeta\right\} & \leq \mathbb{E}\left[F\left(\left|\widetilde{\Delta}\left(t \wedge T \wedge S_{\delta_{0}} \wedge \tau_{R} \wedge \zeta\right)\right|\right) \mathbf{1}_{\left\{S_{\delta_{0}}<t \wedge T \wedge \tau_{R} \wedge \zeta\right\}}\right] \\
& \leq \mathbb{E}\left[F\left(\left|\widetilde{\Delta}\left(t \wedge T \wedge S_{\delta_{0}} \wedge \tau_{R} \wedge \zeta\right)\right|\right)\right] \\
& \leq G^{-1}\left(G \circ F(|x-z|)+2 \kappa_{R} t\right) .
\end{aligned}
$$

This implies that 


$$
\begin{aligned}
\mathbb{P}\left\{S_{\delta_{0}}<t \wedge T \wedge \tau_{R} \wedge \zeta\right\} & \leq \frac{G^{-1}\left(G \circ F(|x-z|)+2 \kappa_{R} t\right)}{F\left(\delta_{0}\right)} \\
& =\frac{1+\delta_{0}}{\delta_{0}} G^{-1}\left(G \circ F(|x-z|)+2 \kappa_{R} t\right) .
\end{aligned}
$$

For any $t \geq 0$ and $\varepsilon>0$, since $\lim _{R \rightarrow \infty} \tau_{R}=\infty$ a.s., we can choose $R>0$ sufficiently large so that

$$
\mathbb{P}\left(t \wedge \zeta>\tau_{R}\right) \leq \mathbb{P}\left(t>\tau_{R}\right)<\varepsilon .
$$

Then it follows that

$$
\begin{aligned}
\mathbb{E} & {[F(|\widetilde{\Delta}(t \wedge \zeta)|)] } \\
= & \mathbb{E}\left[F\left(\left|\widetilde{\Delta}\left(t \wedge \zeta \wedge \tau_{R}\right)\right|\right) \mathbf{1}_{\left\{t \wedge \zeta \leq \tau_{R}\right\}}\right]+\mathbb{E}\left[F(|\widetilde{\Delta}(t \wedge \zeta)|) \mathbf{1}_{\left\{t \wedge \zeta>\tau_{R}\right\}}\right] \\
\leq & \mathbb{E}\left[F\left(\left|\widetilde{\Delta}\left(t \wedge \zeta \wedge T \wedge \tau_{R}\right)\right|\right)\right]+\varepsilon \\
= & \mathbb{E}\left[F\left(\left|\widetilde{\Delta}\left(t \wedge \zeta \wedge T \wedge \tau_{R}\right)\right|\right) \mathbf{1}_{\left\{S_{\delta_{0}}<t \wedge T \wedge \tau_{R} \wedge \zeta\right\}}\right] \\
& +\mathbb{E}\left[F\left(\left|\widetilde{\Delta}\left(t \wedge \zeta \wedge T \wedge \tau_{R}\right)\right|\right) \mathbf{1}_{\left\{S_{\delta_{0}} \geq t \wedge T \wedge \tau_{R} \wedge \zeta\right\}}\right]+\varepsilon \\
\leq & \mathbb{P}\left\{S_{\delta_{0}}<t \wedge T \wedge \tau_{R} \wedge \zeta\right\}+\mathbb{E}\left[F\left(\left|\widetilde{\Delta}\left(t \wedge T \wedge \tau_{R} \wedge S_{\delta_{0}} \wedge \zeta\right)\right|\right)\right]+\varepsilon \\
\leq & \frac{1+2 \delta_{0}}{\delta_{0}} G^{-1}\left(G \circ F(|x-z|)+2 \kappa_{R} t\right)+\varepsilon .
\end{aligned}
$$

Passing to the limit, we obtain

$$
\lim _{x \rightarrow z \rightarrow 0} \mathbb{E}[F(|\widetilde{\Delta}(t \wedge \zeta)|)] \leq 0+\varepsilon=\varepsilon
$$

Since $\varepsilon>0$ is arbitrary, it follows that $\lim _{x-z \rightarrow 0} \mathbb{E}[F(|\widetilde{X}(t \wedge \zeta)-\widetilde{Z}(t \wedge \zeta)|)]=0$.

Choose $R>0$ as in (56). Then we use (51) and (57) to compute

$$
\begin{aligned}
\mathbb{P}\{\zeta \leq t\} & =\mathbb{P}\left\{\zeta \leq t, \tau_{R}<t\right\}+\mathbb{P}\left\{\zeta \leq t, \tau_{R} \geq t\right\} \\
& \leq \mathbb{P}\left\{\tau_{R}<t\right\}+\mathbb{E}\left[\mathbf{1}_{\left\{\widetilde{\Lambda}\left(t \wedge \zeta \wedge \tau_{R}\right) \neq \widetilde{\Xi}\left(t \wedge \zeta \wedge \tau_{R}\right)\right\}}\right] \\
& <\varepsilon+\mathbb{E}\left[g\left(\widetilde{X}\left(t \wedge \zeta \wedge \tau_{R}\right), \widetilde{\Lambda}\left(t \wedge \zeta \wedge \tau_{R}\right), \widetilde{Z}\left(t \wedge \zeta \wedge \tau_{R}\right), \widetilde{\Xi}\left(t \wedge \zeta \wedge \tau_{R}\right)\right)\right] \\
& =\varepsilon+\mathbb{E}\left[\int_{0}^{t \wedge \zeta \wedge \tau_{R}} \widetilde{\mathscr{A} g}(\widetilde{X}(s), \widetilde{\Lambda}(s), \widetilde{Z}(s), \widetilde{\Xi}(s)) \mathrm{d} s\right] \\
& \leq \varepsilon+\mathbb{E}\left[\int_{0}^{t \wedge \zeta \wedge \tau_{R}} \kappa_{R} \rho(F(|\widetilde{\Delta}(s)|)) \mathrm{d} s\right] \\
& \leq \varepsilon+\mathbb{E}\left[\int_{0}^{t \wedge \tau_{R}} \kappa_{R} \rho(F(|\widetilde{\Delta}(s \wedge \zeta)|)) \mathrm{d} s\right] \\
& \leq \varepsilon+\mathbb{E}\left[\int_{0}^{t} \kappa_{R} \rho(F(|\widetilde{\Delta}(s \wedge \zeta)|)) \mathrm{d} s\right] \\
& \leq \varepsilon+\kappa_{R} \int_{0}^{t} \rho(\mathbb{E}[F(|\widetilde{\Delta}(s \wedge \zeta)|)]) \mathrm{d} s
\end{aligned}
$$




$$
\begin{aligned}
& \leq \varepsilon+\kappa_{R} \int_{0}^{t} \rho\left(\frac{1+2 \delta_{0}}{\delta_{0}} G^{-1}\left(G \circ F(|x-z|)+2 \kappa_{R} s\right)+\varepsilon\right) \mathrm{d} s \\
& \leq \varepsilon+\kappa_{R} t \rho\left(\frac{1+2 \delta_{0}}{\delta_{0}} G^{-1}\left(G \circ F(|x-z|)+2 \kappa_{R} t\right)+\varepsilon\right) .
\end{aligned}
$$

Passing to the limit as $x-z \rightarrow 0$, we obtain

$$
\limsup _{x \rightarrow z \rightarrow 0} \mathbb{P}\{\zeta \leq t\} \leq \varepsilon+\kappa_{R} t \rho(\varepsilon) .
$$

Finally, we combine (58) and (59) to obtain

$$
\begin{aligned}
\mathbb{E} & {[f(\widetilde{X}(t), \widetilde{\Lambda}(t), \widetilde{Z}(t), \widetilde{\Xi}(t))] } \\
& =\mathbb{E}\left[F(|\widetilde{X}(t)-\widetilde{Z}(t)|)+\mathbf{1}_{\{\widetilde{\Lambda}(t) \neq \widetilde{\Xi}(t)\}}\right] \\
& =\mathbb{E}\left[F(|\widetilde{X}(t)-\widetilde{Z}(t)|) \mathbf{1}_{\{\zeta>t\}}+F(|\widetilde{X}(t)-\widetilde{Z}(t)|) \mathbf{1}_{\{\zeta \leq t\}}+\mathbf{1}_{\{\widetilde{\Lambda}(t) \neq \widetilde{\Xi}(t)\}}\right] \\
& \leq \mathbb{E}[F(|\widetilde{X}(t \wedge \zeta)-\widetilde{Z}(t \wedge \zeta)|)]+2 \mathbb{P}\{\zeta \leq t\} \\
& \rightarrow \varepsilon+2\left(\varepsilon+\kappa_{R} t \rho(\varepsilon)\right), \text { as }|x-z| \rightarrow 0 .
\end{aligned}
$$

Since $\varepsilon>0$ is arbitrary and $\lim _{r \downarrow 0} \rho(r)=0$, it follows that

$$
\lim _{x \rightarrow z \rightarrow 0} \mathbb{E}[f(\widetilde{X}(t), \widetilde{\Lambda}(t), \widetilde{Z}(t), \widetilde{\Xi}(t))]=0 .
$$

Recall that $f$ is a bounded metric on $\mathbb{R}^{d} \times \mathbb{S}$. Hence it follows that

$$
W_{f}(P(t, x, k, \cdot), P(t, z, k, \cdot)) \leq \mathbb{E}[f(\widetilde{X}(t), \widetilde{\Lambda}(t), \widetilde{Z}(t), \widetilde{\Xi}(t))] \rightarrow 0 \text { as } x \rightarrow z,
$$

where for two probability measures $\mu$ and $v$ on $\mathbb{R}^{d} \times \mathbb{S}$, the Wasserstein distance $W_{f}(\mu, v)$ is defined as

$$
W_{f}(\mu, v):=\inf \left\{\sum_{i, j \in \mathbb{S}} \int f(x, i, y, j) \pi(\mathrm{d} x, i, \mathrm{~d} y, j), \pi \in \mathscr{C}(\mu, v)\right\},
$$

here $\mathscr{C}(\mu, v)$ is the collection of coupling measures for $\mu$ and $v$. Therefore the desired Feller property follows from Theorem 5.6 of Chen (2004).

\section{Strong Feller Property}

Assumption 4.1 For each $k \in \mathbb{S}$ and $x \in \mathbb{R}^{d}$, the stochastic differential equation (27) has a non-exploding weak solution $X^{(k)}$ with initial condition $x$ and the solution is unique in the sense of probability law.

Assumption 4.2 The process $X^{(k)}$ is strong Feller. 
Assumption 4.3 Assume that

$$
H:=\sup \left\{q_{k}(x): x \in \mathbb{R}^{d}, k \in \mathbb{S}\right\}<\infty,
$$

and that there exists a positive constant $\kappa$ such that

$$
0 \leq q_{k l}(x) \leq \kappa l 3^{-l} \text { for all } x \in \mathbb{R}^{d} \text { and } k \neq l \in \mathbb{S} .
$$

Let us briefly comment on the above assumptions. The existence and uniqueness of weak solution to (27) is related to the study of martingale problem for Lévy type operators; see, for example, Komatsu (1973) and Stroock (1975). Condition (60) in Assumption 4.2 is stronger than (10) in Assumption 2.1. We need such a uniform bound in (60) so that we can establish the series representation for the resolvent of the regime-switching jump diffusion $(X, \Lambda)$ in Lemma 4.7, which, in turn, helps to establish the strong Feller property for $(X, \Lambda)$. In general one can obtain the strong Feller property for $X^{(k)}$ under suitable non-degenerate conditions (Kunita (2013)) and certain regularity conditions such as (local) Lipschitz conditions of the coefficients. The following non-Lipschitz sufficient condition for strong Feller property was established in Xi and Zhu (2018a).

Lemma 4.4 Suppose that Assumptions 4.1 holds. In addition, for any given $k \in \mathbb{S}$, suppose that for each $R>0$, there exist positive constants $\lambda_{R}$ and $\kappa_{R}$ such that for all $x, z \in \mathbb{R}^{d}$ with $|x| \vee|z| \leq R$, we have

$$
\langle\xi, a(x, k) \xi\rangle \geq \lambda_{R}|\xi|^{2}, \quad \forall \xi \in \mathbb{R},
$$

and

$$
\begin{aligned}
& \int_{U}\left[|c(x, k, u)-c(z, k, u)|^{2} \wedge(4|x-z| \cdot|c(x, k, u)-c(z, k, u)|)\right] v(\mathrm{~d} u) \\
& +2\langle x-z, b(x, k)-b(z, k)\rangle+\left|\sigma_{\lambda_{R}}(x, k)-\sigma_{\lambda_{R}}(z, k)\right|^{2} \leq 2 \kappa_{R}|x-z| \vartheta(|x-z|)
\end{aligned}
$$

whenever $|x-z| \leq \delta_{0}$, where $\delta_{0}$ is a positive constant, $\vartheta$ is a nonnegative function defined on $\left[0, \delta_{0}\right]$ satisfying $\lim _{r \rightarrow 0} \vartheta(r)=0$, and $\sigma_{\lambda_{R}}(x, k)$ is the unique symmetric nonnegative definite matrix-valued function such that $\sigma_{\lambda_{R}}(x, k)^{2}=a(x, k)-\lambda_{R} I$. Then the process $X^{(k)}$ of (27) is strong Feller continuous.

Next for each $(x, k) \in \mathbb{R}^{d} \times \mathbb{S}$, as in (Øksendal, 2003, Section 8.2), we kill the process $X^{(k)}$ at rate $\left(-q_{k k}\right)$ :

$$
\begin{aligned}
\mathbb{E}_{k}\left[f\left(\widetilde{X}_{x}^{(k)}(t)\right)\right] & =\mathbb{E}_{k}\left[f\left(X_{x}^{(k)}(t)\right) \exp \left\{\int_{0}^{t} q_{k k}\left(X_{x}^{(k)}(s)\right) \mathrm{d} s\right\}\right] \\
& =\mathbb{E}^{(x, k)}\left[t<\tau ; f\left(X^{(k)}(t)\right)\right], \quad f \in \mathfrak{B}_{b}\left(\mathbb{R}^{d}\right),
\end{aligned}
$$

to get a subprocess $\widetilde{X}^{(k)}$, where $\tau:=\inf \{t \geq 0: \Lambda(t) \neq \Lambda(0)\}$. Equivalently, $\widetilde{X}^{(k)}$ can be defined as $\widetilde{X}^{(k)}(t)=X^{(k)}(t)$ if $t<\tau$ and $\widetilde{X}^{(k)}(t)=\partial$ if $t \geq \tau$, where $\partial$ is a cemetery point or a coffin state added to $\mathbb{R}^{d}$ as in (Øksendal, 2003, p. 145). Note that in the 
above, to get the killed process $\widetilde{X}^{(k)}$ from the original process $X^{(k)}$, the killing rate is just the jumping rate of $\Lambda$ from state $k$. Namely, the killing time is just the first switching time $\tau$. To proceed, we denote the transition probability families of the process $X^{(k)}$ and the killed process $\widetilde{X}^{(k)}$ by $\left\{P^{(k)}(t, x, A): t \geq 0, x \in \mathbb{R}^{d}, A \in \mathfrak{B}\left(\mathbb{R}^{d}\right)\right\}$ and $\left\{\widetilde{P}^{(k)}(t, x, A): t \geq 0, x \in \mathbb{R}^{d}, A \in \mathfrak{B}\left(\mathbb{R}^{d}\right)\right\}$, respectively.

Lemma 4.5 Under Assumptions 4.1, 4.2, and 4.3, for each $k \in \mathbb{S}$, the killed process $\widetilde{X}^{(k)}$ has strong Feller property.

Proof. Let $\left\{P_{t}^{(k)}\right\}$ and $\left\{\widetilde{P}_{t}^{(k)}\right\}$ denote the transition semigroups of $X^{(k)}$ and $\widetilde{X}^{(k)}$, respectively. To prove the strong Feller property $\widetilde{X}^{(k)}$, we need only prove that for any given bounded measurable function $f$ on $\mathbb{R}^{d}, \widetilde{P}_{t}^{(k)} f(z)$ is continuous with respect to $z$ for all $t>0$. To this end, for fixed $t>0$ and $0<s<t$, set $g_{s}(z):=\widetilde{P}_{t-s}^{(k)} f(z)$. Clearly, the function $g_{s}(\cdot)$ is bounded and measurable, see the Corollary to Theorem 1.1 in Chung and Zhao (1995). By the strong Feller property of $X^{(k)}, P_{s}^{(k)} g_{s} \in C_{b}\left(\mathbb{R}^{d}\right)$.

To proceed, by the Markov property, we have that

$$
\begin{aligned}
\widetilde{P}_{t}^{(k)} f(x)= & \mathbb{E}_{k}^{(x)}\left[f\left(X^{(k)}(t)\right) \exp \left\{\int_{0}^{t} q_{k k}\left(X^{(k)}(u)\right) \mathrm{d} u\right\}\right] \\
=\mathbb{E}_{k}^{(x)} & {\left[\exp \left\{\int_{0}^{s} q_{k k}\left(X^{(k)}(u)\right) \mathrm{d} u\right\}\right.} \\
& \left.\times \mathbb{E}_{k}^{\left(X^{(k)}(s)\right)}\left[f\left(X^{(k)}(t-s)\right) \exp \left\{\int_{0}^{t-s} q_{k k}\left(X^{(k)}(u)\right) \mathrm{d} u\right\}\right]\right] .
\end{aligned}
$$

Meanwhile, we also have that

$$
\begin{aligned}
P_{s}^{(k)} g_{s}(x) & =P_{s}^{(k)} \widetilde{P}_{t-s}^{(k)} f(x)=\mathbb{E}_{k}^{(x)}\left[\widetilde{P}_{t-s}^{(k)} f\left(X^{(k)}(s)\right)\right] \\
& =\mathbb{E}_{k}^{(x)}\left[\mathbb{E}_{k}^{\left(X^{(k)}(s)\right)}\left[f\left(X^{(k)}(t-s)\right) \exp \left\{\int_{0}^{t-s} q_{k k}\left(X^{(k)}(u)\right) \mathrm{d} u\right\}\right]\right] .
\end{aligned}
$$

Recall from Assumption 4.2 that $+\infty>H \geq-\inf \left\{q_{k k}(x):(x, k) \in \mathbb{R}^{2 d} \times \mathbb{S}\right\}$ and $q_{k k}(x) \leq 0$, and so

$$
0 \leq 1-\exp \left\{\int_{0}^{s} q_{k k}\left(X^{(k)}(u)\right) \mathrm{d} u\right\} \leq\left(1-e^{-H s}\right) .
$$

Thus, it follows from (63), (64) and (65) that

$$
\left|P_{s}^{(k)} g_{s}(x)-\widetilde{P}_{t}^{(k)} f(x)\right| \leq\left(1-e^{-H s}\right)\|f\| \rightarrow 0 \text { uniformly as } s \rightarrow 0,
$$

where $\|\cdot\|$ denotes the uniform (or supremum) norm. Combining this with the fact that $P_{s}^{(k)} g_{s} \in C_{b}\left(\mathbb{R}^{d}\right)$ implies that $\widetilde{P}_{t}^{(k)} f \in C_{b}\left(\mathbb{R}^{d}\right)$, and so the desired strong Feller property follows.

The following lemma was proved in Xi and Zhu (2018b). 
Lemma 4.6 Let $\Xi$ be a right continuous strong Markov process and $q: \mathbb{R}^{d} \mapsto \mathbb{R} a$ nonnegative bounded measurable function. Denote by $\widetilde{\Xi}$ the subprocess of $\Xi$ killed at rate $q$ with lifetime $\zeta$ :

$$
\mathbb{E}\left[f\left(\widetilde{\Xi}^{(z)}(t)\right)\right]:=\mathbb{E}\left[t<\zeta ; f\left(\Xi^{(z)}(t)\right)\right]=\mathbb{E}\left[f\left(\Xi^{(z)}(t)\right) \exp \left\{-\int_{0}^{t} q\left(\Xi^{(z)}(s)\right) \mathrm{d} s\right\}\right] .
$$

Then for any constant $\alpha>0$ and nonnegative function $\phi$ on $\mathbb{R}^{d}$, we have

$$
\mathbb{E}\left[e^{-\alpha \zeta} \phi(\widetilde{\Xi}(z)(\zeta-))\right]=G_{\alpha}^{\widetilde{\Xi}}(q \phi)(z),
$$

where $\left\{G_{\alpha}^{\widetilde{\Xi}}, \alpha>0\right\}$ denotes the resolvent for the killed process $\widetilde{\Xi}$.

For each $k \in \mathbb{S}$, let $\left\{\widetilde{G}_{\alpha}^{(k)}, \alpha>0\right\}$ be the resolvent for the generator $\mathscr{L}_{k}+q_{k k}$. Denote by $\left\{G_{\alpha}, \alpha>0\right\}$ the resolvent for the generator $\mathscr{A}$ defined in (5). Let

$$
\widetilde{G}_{\alpha}=\left(\begin{array}{cccc}
\widetilde{G}_{\alpha}^{(1)} & 0 & 0 & \ldots \\
0 & \widetilde{G}_{\alpha}^{(2)} & 0 & \ldots \\
0 & 0 & \widetilde{G}_{\alpha}^{(3)} & \ldots \\
\vdots & \vdots & \vdots & \ddots
\end{array}\right) \text { and } Q^{0}(x)=Q(x)-\left(\begin{array}{cccc}
q_{11}(x) & 0 & 0 & \ldots \\
0 & q_{22}(x) & 0 & \ldots \\
0 & 0 & q_{33}(x) & \ldots \\
\vdots & \vdots & \vdots & \ddots
\end{array}\right)
$$

Lemma 4.7 Suppose that Assumptions 4.1, 4.2, and 4.3 hold. Then there exists a constant $\alpha_{1}>0$ such that for any $\alpha \geq \alpha_{1}$ and any $f(\cdot, k) \in \mathfrak{B}_{b}\left(\mathbb{R}^{d}\right)$ with $k \in \mathbb{S}$,

$$
G_{\alpha} f=\widetilde{G}_{\alpha} f+\sum_{m=1}^{\infty} \widetilde{G}_{\alpha}\left(Q^{0} \widetilde{G}_{\alpha}\right)^{m} f
$$

Proof. Let $f(z, k) \geq 0$ on $\mathbb{R}^{2 d} \times \mathbb{S}$. Applying the strong Markov property at the first switching time $\tau$ and recalling the construction of $(Z, \Lambda)$, we obtain

$$
\begin{aligned}
G_{\alpha} f(z, k) & =\mathbb{E}_{z, k}\left[\int_{0}^{\infty} e^{-\alpha t} f(Z(t), \Lambda(t)) \mathrm{d} t\right] \\
& =\mathbb{E}_{z, k}\left[\int_{0}^{\tau} e^{-\alpha t} f(Z(t), k) \mathrm{d} t\right]+\mathbb{E}_{z, k}\left[\int_{\tau}^{\infty} e^{-\alpha t} f(Z(t), \Lambda(t)) \mathrm{d} t\right] \\
& =\widetilde{G}_{\alpha}^{(k)} f(z, k)+\mathbb{E}_{z, k}\left[e^{-\alpha \tau} G_{\alpha} f(Z(\tau), \Lambda(\tau))\right] \\
& =\widetilde{G}_{\alpha}^{(k)} f(z, k)+\sum_{l \in \mathbb{S} \backslash\{k\}} \mathbb{E}_{z, k}\left[e^{-\alpha \tau}\left(-\frac{q_{k l}}{q_{k k}}\right)(Z(\tau-)) G_{\alpha} f(Z(\tau-), l)\right] \\
& =\widetilde{G}_{\alpha}^{(k)} f(z, k)+\sum_{l \in \mathbb{S} \backslash\{k\}} \widetilde{G}_{\alpha}^{(k)}\left(q_{k l} G_{\alpha} f(\cdot, l)\right)(z),
\end{aligned}
$$

where the last equality follows from (68) in Lemma 4.6. Hence we have 


$$
G_{\alpha} f(z, k)=\widetilde{G}_{\alpha}^{(k)} f(\cdot, k)(z)+\widetilde{G}_{\alpha}^{(k)}\left(\sum_{l \in \mathbb{S} \backslash\{k\}} q_{k l} G_{\alpha} f(\cdot, l)\right)(z) .
$$

Repeating the above argument, the second term on the right-hand side of (70) equals $\widetilde{G}_{\alpha}^{(k)}\left(\sum_{l \in \mathbb{S} \backslash\{k\}} q_{k l} \widetilde{G}_{\alpha}^{(l)} f(\cdot, l)\right)(z)+\widetilde{G}_{\alpha}^{(k)}\left(\sum_{l \in \mathbb{S} \backslash\{k\}} q_{k l} \widetilde{G}_{\alpha}^{(l)}\left(\sum_{l_{1} \in \mathbb{S} \backslash\{l\}} q_{l l_{1}} G_{\alpha} f\left(\cdot, l_{1}\right)\right)\right)(z)$.

Hence, we further obtain that for any fixed $k \in \mathbb{S}$ and any integer $m \geq 1$,

$$
G_{\alpha} f(z, k)=\sum_{i=0}^{m} \psi_{i}^{(k)}(z)+R_{m}^{(k)}(z)
$$

where

$$
\begin{aligned}
& \psi_{0}^{(k)}=\widetilde{G}_{\alpha}^{(k)} f(\cdot, k), \\
& \psi_{1}^{(k)}=\widetilde{G}_{\alpha}^{(k)}\left(\sum_{l \in \mathbb{S} \backslash\{k\}} q_{k l} \widetilde{G}_{\alpha}^{(l)} f(\cdot, l)\right)=\widetilde{G}_{\alpha}^{(k)}\left(\sum_{l \in \mathbb{S} \backslash\{k\}} q_{k l} \psi_{0}^{(l)}\right), \\
& \psi_{i}^{(k)}=\widetilde{G}_{\alpha}^{(k)}\left(\sum_{l \in \mathbb{S} \backslash\{k\}} q_{k l} \psi_{i-1}^{(l)}\right) \text { for } i \geq 1,
\end{aligned}
$$

and

$$
\begin{aligned}
R_{m}^{(k)}=\widetilde{G}_{\alpha}^{(k)}\left(\sum_{l_{1} \in \mathbb{S} \backslash\{k\}} q_{k, l_{1}} \widetilde{G}_{\alpha}^{\left(l_{1}\right)}\right. & \left(\sum _ { l _ { 2 } \in \mathbb { S } \backslash \{ l _ { 1 } \} } q _ { l _ { 1 } , l _ { 2 } } \widetilde { G } _ { \alpha } ^ { ( l _ { 2 } ) } \left(\ldots \left(\sum_{l_{m-1} \in \mathbb{S} \backslash\left\{l_{m-2}\right\}} q_{l_{m-2}, l_{m-1}}\right.\right.\right. \\
& \left.\left.\left.\left.\widetilde{G}_{\alpha}^{\left(l_{m-1}\right)}\left(\sum_{l_{m} \in \mathbb{S} \backslash\left\{l_{m-1}\right\}} q_{l_{m-1}, l_{m}} G_{\alpha} f\left(\cdot, l_{m}\right)\right)\right)\right)\right)\right) .
\end{aligned}
$$

We have

$$
\left\|\psi_{0}^{(k)}\right\|=\left\|\mathbb{E}_{\cdot, k}\left[\int_{0}^{\tau} e^{-\alpha t} f(Z(t), k) \mathrm{d} t\right]\right\| \leq \frac{\|f\|}{\alpha} .
$$

Note that the same calculation reveals that (72) in fact holds for all $l \in \mathbb{S},\left\|\psi_{0}^{(l)}\right\| \leq$ $\frac{\|f\|}{\alpha}$. Thanks to Assumption 4.3, $q_{k l}(z) \leq \frac{\kappa l}{3 l}$ for all $l \neq k$ and $x \in \mathbb{R}^{d}$. Consequently, we can compute

$$
\begin{aligned}
\left\|\psi_{1}^{(k)}\right\| \leq \sum_{l \in \mathbb{S} \backslash\{k\}}\left\|\widetilde{G}_{\alpha}^{(k)}\left(q_{k l} \psi_{0}^{(l)}\right)\right\| & \leq \sum_{l \in \mathbb{S} \backslash\{k\}} \frac{\kappa l}{3^{l}} \cdot \frac{\left\|\psi_{0}^{(l)}\right\|}{\alpha} \\
& \leq \sum_{l \in \mathbb{S} \backslash\{k\}} \frac{\kappa l}{3^{l}} \cdot \frac{\|f\|}{\alpha^{2}}=\frac{3 \kappa}{4 \alpha} \cdot \frac{\|f\|}{\alpha} .
\end{aligned}
$$


As before, we observe that (73) actually holds for all $l \in \mathbb{S}$. Similarly, we can use induction to show that

$$
\left\|\psi_{i}^{(k)}\right\| \leq\left(\frac{3 \kappa}{4 \alpha}\right)^{i} \cdot \frac{\|f\|}{\alpha}, \quad \text { for } i \geq 2
$$

and

$$
\left\|R_{m}^{(k)}\right\| \leq\left(\frac{3 \kappa}{4 \alpha}\right)^{m+1} \cdot \frac{\|f\|}{\alpha} .
$$

Now let $\alpha_{1}:=\frac{3 \kappa+1}{4}$ and $\alpha \geq \alpha_{1}$. Then we have for each $k \in \mathbb{S}, G_{\alpha} f(\cdot, k)=\sum_{i=0}^{\infty} \psi_{i}^{(k)}$, which clearly implies (69). The lemma is proved.

Lemma 4.7 establishes an explicit relationship of the resolvents for $(Z, \Lambda)$ and the killed processes $\widetilde{Z}^{(k)}, k \in \mathbb{S}$. This, together with the strong Feller property for the killed processes $\widetilde{Z}^{(k)}, k \in \mathbb{S}$ (Lemma 4.5), enables us to derive the strong Feller property for $(Z, \Lambda)$ in the following theorem.

Theorem 4.8 Suppose that Assumptions 3.1, 4.1, 4.2, and 4.3 hold. Then the process $(X, \Lambda)$ has the strong Feller property.

Proof. The proof is almost identical to that of Theorem 5.4 in Xi and Zhu (2018b) and for brevity, we shall only give a sketch here. Denote the transition probability family of Markov process $(X, \Lambda)$ by $\left\{P(t,(x, k), A): t \geq 0,(x, k) \in \mathbb{R}^{d} \times \mathbb{S}, A \in\right.$ $\left.\mathscr{B}\left(\mathbb{R}^{d} \times \mathbb{S}\right)\right\}$. Then it follows from Lemma 4.7 that

$$
\begin{aligned}
& P(t,(x, k), A \times\{l\})=\delta_{k l} \widetilde{P}^{(k)}(t, x, A) \\
& \quad+\sum_{m=1}^{+\infty} \int_{0<t_{1}<\cdots<t_{m}<t} \cdots \int_{\substack{l_{1} \in \mathbb{S} \backslash\left\{l_{0}\right\}, l_{2} \in \mathbb{S} \backslash\left\{l_{1}\right\}, \ldots, l_{m} \in \mathbb{S} \backslash\left\{l_{m-1}\right\} \\
l_{0}=k, l_{m}=l}} \int_{\mathbb{R}^{d}} \cdots \int_{\mathbb{R}^{d}} \widetilde{P}^{\left(l_{0}\right)}\left(t_{1}, x, \mathrm{~d} x_{1}\right) \\
& \quad \times q_{l_{0} l_{1}}\left(x_{1}\right) \widetilde{P}^{\left(l_{1}\right)}\left(t_{2}-t_{1}, x_{1}, \mathrm{~d} x_{2}\right) \cdots q_{l_{m-1} l_{m}}\left(x_{m}\right) \widetilde{P}^{\left(l_{m}\right)}\left(t-t_{m}, x_{m}, A\right) \mathrm{d} t_{1} \mathrm{~d} t_{2} \ldots \mathrm{d} t_{m},
\end{aligned}
$$

where $\delta_{k l}$ is the Kronecker symbol in $k, l$, which equals 1 if $k=l$ and 0 if $k \neq l$. By Lemma 4.5 , we know that for every $k \in \mathbb{S}, \widetilde{X}^{(k)}$ has the strong Feller property. Therefore, in view of Proposition 6.1.1 in Meyn and Tweedie (1993), we derive that $\widetilde{P}^{(k)}(t, x, A)$ and every term in the series on the right-hand side of (76) are lower semicontinuous with respect to $x$ whenever $A$ is an open set in $\mathfrak{B}\left(\mathbb{R}^{d}\right)$. Note that $\mathbb{S}$ is a countably infinite set and has discrete metric. Therefore it follows that the left-hand side of (76) is lower semicontinuous with respect to $(x, k)$ for every $l \in \mathbb{S}$ whenever $A$ is an open set in $\mathfrak{B}\left(\mathbb{R}^{d}\right)$. Consequently, $(X, \Lambda)$ has the strong Feller property (see Proposition 6.1.1 in Meyn and Tweedie (1993) again). The theorem is proved.

Remark 4.9 Shao (2015) proves that for a state-independent regime-switching diffusion processes, the strong Feller property for each subdiffusion implies the strong Feller property for regime-switching diffusion processes. This work further proves this implication for state-dependent regime-switching jump diffusion processes. 
Remark 4.10 The strong Feller property for regime-switching jump diffusions was also studied in $\mathrm{Xi}$ and Zhu (2017), where it is assumed that $v(U)<\infty$ is a finite measure, i.e., the jump part is modeled by a compound Poisson process. In addition, a finite-range condition for the switching component is placed in that paper and is key to the analyses there. Here these two restrictions are removed.

\section{References}

Bass, R. F. (2003). Stochastic differential equations driven by symmetric stable processes. In Séminaire de Probabilités, XXXVI, volume 1801 of Lecture Notes in Math., pages 302-313. Springer, Berlin.

Chen, M.-F. (2004). From Markov chains to non-equilibrium particle systems. World Scientific Publishing Co. Inc., River Edge, NJ, second edition.

Chen, X. Chen, Z.-Q. Tran, K. and Yin, G. (2018a). Properties of switching jump diffusions: Maximum principles and Harnack inequalities, to appear in Bernoulli.

Chen, X. Chen, Z.-Q. Tran, K. and Yin, G. (2018b). Recurrence and ergodicity for a class of regime-switching jump diffusions, to appear in Appl. Math. Optim., https://link.springer.com/article/10.1007/s00245-017-9470-9.

Chung, K. L. and Zhao, Z. X. (1995). From Brownian motion to Schrödinger's equation, volume 312 of Grundlehren der Mathematischen Wissenschaften [Fundamental Principles of Mathematical Sciences]. Springer-Verlag, Berlin.

Fang, S. and Zhang, T. (2005). A study of a class of stochastic differential equations with non-Lipschitzian coefficients. Probab. Theory Related Fields, 132(3):356390.

$\mathrm{Fu}, \mathrm{Z}$. and Li, Z. (2010). Stochastic equations of non-negative processes with jumps. Stochastic Process. Appl., 120(3):306-330.

Klebaner, F. C. (2005). Introduction to stochastic calculus with applications. Imperial College Press, London, second edition.

Komatsu, T. (1973). Markov processes associated with certain integro-differential operators. Osaka J. Math., 10:271-303.

Kunita, H. (2013). Nondegenerate SDE's with jumps and their hypoelliptic properties. J. Math. Soc. Japan, 65(3):993-1035.

Lawley, S. D., Mattingly, J. C., and Reed, M. C. (2014). Sensitivity to switching rates in stochastically switched ODEs. Commun. Math. Sci., 12(7):1343-1352.

Li, Z. and Mytnik, L. (2011). Strong solutions for stochastic differential equations with jumps. Ann. Inst. Henri Poincaré Probab. Stat., 47(4):1055-1067.

$\mathrm{Li}, \mathrm{Z}$. and Pu, F. (2012). Strong solutions of jump-type stochastic equations. Electron. Commun. Probab., 17(33):1-13.

Mao, X. and Yuan, C. (2006). Stochastic differential equations with Markovian switching. Imperial College Press, London.

Meyn, S. P. and Tweedie, R. L. (1993). Markov chains and stochastic stability. Communications and Control Engineering Series. Springer-Verlag London, Ltd., London. 
Nguyen, D. H. and Yin, G. (2016). Modeling and analysis of switching diffusion systems: past-dependent switching with a countable state space. SIAM J. Control Optim., 54(5):2450-2477.

Nguyen, D. H. and Yin, G. (2018). Recurrence and ergodicity of switching diffusions with past-dependent switching having a countable state space. Potential Anal., 48(4):405-435.

Norris, J. R. (1998). Markov chains, volume 2 of Cambridge Series in Statistical and Probabilistic Mathematics. Cambridge University Press, Cambridge. Reprint of 1997 original.

$\varnothing \mathrm{ksendal}$, B. (2003). Stochastic differential equations, An introduction with applications. Universitext. Springer-Verlag, Berlin, sixth edition.

Shao, J. (2015). Strong solutions and strong Feller properties for regimeswitching diffusion processes in an infinite state space. SIAM J. Control Optim., 53(4):2462-2479.

Stroock, D. W. (1975). Diffusion processes associated with Lévy generators. Z. Wahrscheinlichkeitstheorie und Verw. Gebiete, 32(3):209-244.

Xi, F. and Zhu, C. (2017). On Feller and strong Feller properties and exponential ergodicity of regime-switching jump diffusion processes with countable regimes. SIAM J. Control Optim., 55(3):1789-1818.

Xi, F. and Zhu, C. (2018a). Jump type stochastic differential equations with nonlipschitz coefficients: Non confluence, feller and strong feller properties, and exponential ergodicity. J. Differential Equations, to appear.

Xi, F. and Zhu, C. (2018b). On the martingale problem and Feller and strong Feller properties for weakly coupled Lévy type operators. Stochastic Process. Appl., to appear.

Yamada, T. and Watanabe, S. (1971). On the uniqueness of solutions of stochastic differential equations. J. Math. Kyoto Univ., 11:155-167.

Yin, G., Zhao, G., and Wu, F. (2012). Regularization and stabilization of randomly switching dynamic systems. SIAM J. Appl. Math., 72(5):1361-1382.

Yin, G. G. and Zhu, C. (2010). Hybrid Switching Diffusions: Properties and Applications, volume 63 of Stochastic Modelling and Applied Probability. Springer, New York. 